\title{
EXTINCTION PROBABILITY OF INTERACTING BRANCHING COLLISION PROCESSES
}

\author{
ANYUE CHEN, ${ }^{* *}$ University of Liverpool and Xian Jiaotong-Liverpool University \\ JUNPING LI, ${ }^{* * *}$ Central South University \\ YIQING CHEN, ${ }^{* * * * *}$ University of Liverpool \\ DINGXUAN ZHOU, ${ }^{* * * * *}$ City University of Hong Kong
}

\begin{abstract}
We consider the uniqueness and extinction properties of the interacting branching collision process (IBCP), which consists of two strongly interacting components: an ordinary Markov branching process and a collision branching process. We establish that there is a unique IBCP, and derive necessary and sufficient conditions for it to be nonexplosive that are easily checked. Explicit expressions are obtained for the extinction probabilities for both regular and irregular cases. The associated expected hitting times are also considered. Examples are provided to illustrate our results.
\end{abstract}

Keywords: Markov branching process; collision branching process; interaction; uniqueness; regularity; extinction probability; extinction time

2010 Mathematics Subject Classification: Primary 60J27

Secondary 60J35

\section{Introduction}

The primary aim of this paper is to tackle a very interesting as well as challenging open problem regarding the extinction probability of the interacting branching collision process (defined below), which is an important class of interacting branching system. As is well known, there has been an extensive interest in generalizing the ordinary Markov branching processes (MBPs) into more general interacting branching models. Such increasing interest is mainly due to the fact that the basic property which governs the evolution of an MBP (i.e. different particles act independently) is not appropriate in many real situations. Indeed, in real situations, particularly in biological science, individuals (particles) usually interact with each other.

The interacting branching collision process, as one of the most important subclasses of interacting branching systems, consists of two strongly interacting components. The first component is an ordinary $\mathrm{MBP}$ and the other is a collision branching process (CBP). For the former, see the good references of Harris [8], Athreya and Ney [4], Asmussen and Hering [2], and Athreya and Jagers [3], whilst, for the latter, see Kalinkin [9], [10], Chen et al. [5], [6], Lange [11], and the references therein. Note that, compared to the large number of publications

\footnotetext{
Received 11 May 2010; revision received 11 June 2011.

* Postal address: Department of Mathematical Sciences, University of Liverpool, Liverpool, L69 7ZL, UK.

** Email address: achen@liv.ac.uk

*** Postal address: School of Mathematical Science and Computing Technology, Central South University, Changsha City, Hunan 410075, China. Email address: jpli@mail.csu.edu.cn

**** Email address: yiqing.chen@liv.ac.uk

***** Postal address: Department of Mathematics, City University of Hong Kong, Tat Chee Avenue, Kowloon, Hong Kong. Email address: mazhou@cityu.edu.hk
} 
for the former, there exist much fewer papers in the literature that discuss the latter. This is because in the evolution of a collision branching process, different from an MBP, the branching events are effected by the interaction/collision of pairs of particles, rather than by the particles individually as in an MBP and, thus, the analysis becomes much more difficult. However, many challenging but important and interesting questions have arisen due to such an interaction effect.

The interacting branching collision process is, however, even more challenging and interesting since in addition to the interaction within the CBP, the two components also strongly interact with each other. Hence, investigating the properties of this process is of great significance.

Although little progress has been made to date, the interacting branches collision process has attracted much attention. Interest in this process can be traced back at least to 1982; see [13]. One of the important questions, that of the extinction probabilities, has been addressed in [10] and an explicit expression for the extinction probabilities has been obtained for some special examples. More importantly, some new methods and techniques in tackling these questions have been introduced and applied in [9] and [10]. In particular, in addition to using the Kolmogorov forward equation, the Kolmogorov backward equation and the exponential generating function were used in [9] and [10]. Some further discussions are available in [11].

The main aim of this paper is to consider more general models for such interacting branching collision processes based on the previous research mentioned above. We will focus on discussing the two most basic questions, that of the uniqueness and extinction probabilities, for the general model and resolve some related interesting and important open questions. Our main solution method will use the Kolmogorov forward equation.

The general model we will address in this paper is a continuous-time Markov chain defined on the state space of nonnegative integers $\mathbb{Z}_{+}=\{0,1,2, \ldots\}$, which represents the evolution of some interacting particles. More formally, we define the model by specifying its infinitesimal characteristic, i.e. the so-called $q$-matrix as follows.

Definition 1.1. A $q$-matrix $Q=\left(q_{i j} ; i, j \in \mathbb{Z}_{+}\right)$is called an interacting branching collision $q$-matrix (henceforth referred to as an IBC $q$-matrix) if

$$
q_{i j}= \begin{cases}\left(\begin{array}{l}
i \\
2
\end{array}\right) c_{j-i+2}+i b_{j-i+1} & \text { if } i \geq 1, j \geq i-2 \\
0 & \text { otherwise }\end{cases}
$$

where

$$
\begin{array}{llll}
c_{0}>0, & c_{j} \geq 0 \quad(j \neq 2), & \sum_{k=3}^{\infty} c_{k}>0, & 0<\sum_{j \neq 2} c_{j}=-c_{2}<\infty, \\
b_{0}>0, & b_{j} \geq 0 \quad(j \neq 1), & \sum_{k=2}^{\infty} b_{k}>0, & 0<\sum_{j \neq 1} b_{j}=-b_{1}<\infty,
\end{array}
$$

together with the conventions that $b_{-1}=0$ and $\left(\begin{array}{l}1 \\ 2\end{array}\right)=0$.

Definition 1.2. A Markov interacting branching collision process (henceforth referred to as an IBCP) is a continuous-time Markov chain on the state space $\mathbb{Z}_{+}$whose transition function $P(t)=\left(p_{i j}(t) ; i, j \in \mathbb{Z}_{+}\right)$satisfies

$$
P^{\prime}(t)=P(t) Q
$$

where $Q$ is given in (1.1)-(1.2). 
The structure of this paper is as follows. Some preliminary results are firstly obtained in Section 2. Uniqueness and regularity criteria are then obtained in Section 3. We show that the IBCP is honest, i.e. the infinitesimal $q$-matrix $Q$ is regular, if and only if the mean birth rate is less than or equal to the mean death rate for the CBP component only. We also show that there always exists only one IBCP for a given $q$-matrix $Q$. The important question of the extinction probability together with the mean extinction time is extensively discussed in Sections 4 and 5. The regular case, which is relatively easy, is fully discussed in Section 4, while the very subtle irregular, i.e. the explosive, case is deeply analysed in Section 5. For both cases, the explicit expressions for extinction probabilities are presented. We show that the extinction probabilities will be mainly dominated by the CBP component rather than the MBP component. Finally, in Section 6 a couple of examples are provided to illustrate the results obtained in the previous sections.

\section{Preliminary}

In order to investigate the properties of IBCPs, it is necessary to define the generating functions of the two known sequences $\left\{c_{k} ; k \geq 0\right\}$ and $\left\{b_{k} ; \geq 0\right\}$ as

$$
C(s)=\sum_{k=0}^{\infty} c_{k} s^{k} \quad \text { and } \quad B(s)=\sum_{k=0}^{\infty} b_{k} s^{k} .
$$

These two functions play extremely important roles in our later analysis. It is clear that $C(s)$ and $B(s)$ are well defined at least on $[-1,1]$. The following simple yet important properties of these functions will be constantly used in this paper and we state them here for the sake of convenience. However, their proofs are easy and well known, and thus omitted.

Lemma 2.1. (i) The equation $C(s)=0$ has at most two roots in $[0,1]$ and exactly one root in $[-1,0)$. More specifically, if $C^{\prime}(1) \leq 0$ then $C(s)>0$ for all $s \in[0,1)$ and 1 is the only root of the equation $C(s)=0$ in $[0,1]$, which is simple or with multiplicity 2 according to $C^{\prime}(1)<0$ or $C^{\prime}(1)=0$, while if $0<C^{\prime}(1) \leq+\infty$ then $C(s)=0$ has an additional simple root $\rho_{c}$ satisfying $0<\rho_{c}<1$ such that $C(s)>0$ for $s \in\left(0, \rho_{c}\right)$ and $C(s)<0$ for $s \in\left(\rho_{c}, 1\right)$. Also, $C(s)=0$ has exactly one root, denoted by $\zeta_{c}$, in $[-1,0]$ such that $C(s)>0$ for all $s \in\left(\zeta_{c}, 0\right]$ and $\left|\zeta_{c}\right| \leq \rho_{c}$. This root is simple unless $C^{\prime}(1)=0$ and $\sum_{k=0}^{\infty} c_{2 k+1}=0$. Also, $\left|\zeta_{c}\right|=\rho_{c}$ if and only if $\sum_{k=0}^{\infty} c_{2 k+1}=0$. Moreover, $C(z)=0$ has no other root in the complex $\operatorname{disk}\{z ;|z| \leq 1\}$.

(ii) The equation $B(s)=0$ has at most two roots in $[0,1]$. More specifically, if $B^{\prime}(1) \leq 0$ then $B(s)>0$ for all $s \in[-1,1)$ and 1 is the only root of $B(s)=0$ in $[0,1)$. If $0<B^{\prime}(1) \leq+\infty$ then $B(s)=0$ has an additional root in $[0,1)$, denoted by $\rho_{b}$, such that $B(s)>0$ for all $s \in\left[-1, \rho_{b}\right)$ and $B(s)<0$ for $s \in\left(\rho_{b}, 1\right)$. Moreover, $B(z)=0$ has no other root in the complex disk $\{z ;|z| \leq 1\}$.

Throughout this paper, we will let $\rho_{c}$ and $\rho_{b}$ denote the smallest nonnegative roots of $C(s)=$ 0 and $B(s)=0$, respectively.

Lemma 2.2. Suppose that $Q$ is an IBC q-matrix as defined in (1.1)-(1.2), and let $P(t)=$ $\left(p_{i j}(t) ; i, j \geq 0\right)$ and $\Phi(\lambda)=\left(\phi_{i j}(\lambda) ; i, j \geq 0\right)$ be a $Q$-function and its $Q$-resolvent, respectively. Furthermore, assume that the $Q$-function $P(t)$ and $Q$-resolvent $\Phi(\lambda)$ satisfy the Kolmogorov forward equation (1.3). Then, for any $i \geq 0, t \geq 0, \lambda>0$, and $|s|<1$, 
we have

$$
\frac{\partial F_{i}(t, s)}{\partial t}=\frac{C(s)}{2} \frac{\partial^{2} F_{i}(t, s)}{\partial s^{2}}+B(s) \frac{\partial F_{i}(t, s)}{\partial s}
$$

or, equivalently,

$$
\Phi_{i}(\lambda, s)-s^{i}=\frac{C(s)}{2} \frac{\partial^{2} \Phi_{i}(\lambda, s)}{\partial s^{2}}+B(s) \frac{\partial \Phi_{i}(\lambda, s)}{\partial s},
$$

where $F_{i}(t, s)=\sum_{j=0}^{\infty} p_{i j}(t) s^{j}$ and $\Phi_{i}(\lambda, s)=\sum_{j=0}^{\infty} \phi_{i j}(\lambda) s^{j}$.

Proof. It follows from the Kolmogorov forward equation (1.3) that, for any $i, j \geq 0$,

$$
p_{i j}^{\prime}(t)=\sum_{k=2}^{j+2} p_{i k}(t)\left(\begin{array}{l}
k \\
2
\end{array}\right) c_{j-k+2}+\sum_{k=1}^{j+1} p_{i k}(t) k b_{j-k+1} .
$$

Multiplying both sides of the above equality by $s^{j}$ and summing over $\mathbb{Z}_{+}$we immediately obtain (2.1). Taking the Laplace transform of both sides of (2.1) then yields (2.2).

Lemma 2.3. Suppose that $Q$ is an IBC q-matrix as defined in (1.1)-(1.2). Let $P(t)=$ $\left(p_{i j}(t) ; i, j \geq 0\right)$ be a $Q$-function that satisfies the Kolmogorov forward equations. Then the following statements hold.

(i) $\int_{0}^{\infty} p_{i j}(t) \mathrm{d} t<+\infty(i, j \geq 1)$ and, thus, $\lim _{t \rightarrow \infty} p_{i j}(t)=0(i, j \geq 1)$.

(ii) For any $i \geq 1$ and $s \in[0,1)$,

$$
\sum_{j=1}^{\infty}\left(\int_{0}^{\infty} p_{i j}(t) \mathrm{d} t\right) s^{j}<+\infty
$$

Proof. It is clear that every positive state is transient and, thus, (i) follows. This simple fact can also be easily obtained analytically. Indeed, by the Kolmogorov forward equation we have

$$
p_{i 0}^{\prime}(t)=p_{i 2}(t) c_{0}+p_{i 1}(t) b_{0}, \quad i \geq 1,
$$

which implies that $\int_{0}^{\infty} p_{i 2}(t) \mathrm{d} t<+\infty$ since $c_{0}>0$. Hence, by the irreducibility of positive states we know that $\int_{0}^{\infty} p_{i j}(t) \mathrm{d} t<+\infty$ for all $i, j \geq 1$.

We now prove (2.3). First note that the partial differential equation (2.1) just states that, for any $i \geq 1$ and $|s|<1$, we have

$$
\sum_{j=0}^{\infty} p_{i j}^{\prime}(t) s^{j}=\frac{C(s)}{2} \sum_{k=2}^{\infty} p_{i k}(t) k(k-1) s^{k-2}+B(s) \sum_{k=1}^{\infty} p_{i k}(t) k s^{k-1}
$$

which can be rewritten as

$$
\sum_{j=0}^{\infty} p_{i j}^{\prime}(t) s^{j}=\sum_{k=1}^{\infty}\left[\frac{(k-1) C(s)}{2}+s B(s)\right] p_{i k}(t) k s^{k-2} .
$$

Now, if $C^{\prime}(1) \leq 0$ then by Lemma 2.1 we have $C(\hat{s})>0$ for any $\hat{s} \in[0,1)$. It follows that there exists a $\hat{k} \geq 2$ such that $\frac{1}{2}(k-1) C(\hat{s})+\hat{s} B(\hat{s})>0$ for any $k \geq \hat{k}$. Then by (2.5) we 
obtain

$$
\begin{aligned}
& {\left[\frac{(\hat{k}-1) C(\hat{s})}{2}+\hat{s} B(\hat{s})\right] \sum_{k=\hat{k}}^{\infty} p_{i k}(t) k \hat{s}^{k-2}} \\
& \quad \leq \sum_{j=0}^{\infty} p_{i j}^{\prime}(t) \hat{s}^{j}-\sum_{k=1}^{k-1}\left[\frac{(k-1) C(\hat{s})}{2}+\hat{s} B(\hat{s})\right] p_{i k}(t) k \hat{s}^{k-2} .
\end{aligned}
$$

Integrating the above inequality yields

$$
\begin{aligned}
& {\left[\frac{(\hat{k}-1) C(\hat{s})}{2}+\hat{s} B(\hat{s})\right] \sum_{k=\hat{k}}^{\infty}\left(\int_{0}^{\infty} p_{i k}(t) \mathrm{d} t\right) k \hat{s}^{k-2}} \\
& \quad \leq \lim _{t \rightarrow \infty} p_{i 0}(t)-\hat{s}^{i}-\sum_{k=1}^{\hat{k}-1}\left[\frac{(k-1) C(\hat{s})}{2}+\hat{s} B(\hat{s})\right]\left(\int_{0}^{\infty} p_{i k}(t) \mathrm{d} t\right) k \hat{s}^{k-2} \\
& \quad<+\infty,
\end{aligned}
$$

which implies (2.3).

On the other hand, if $0<C^{\prime}(1) \leq+\infty$ then, again by Lemma 2.1, we know that $C(s)=0$ has a smallest nonnegative root $\rho_{c} \in[0,1)$ such that $C(s)<0$ for any $s \in\left(\rho_{c}, 1\right)$. Now, for any $\hat{s} \in\left(\rho_{c}, 1\right)$, there exists a $\hat{k} \geq 2$ such that $\frac{1}{2}(k-1) C(\hat{s})+\hat{s} B(\hat{s})<0$ for any $k \geq \hat{k}$. Then, again by (2.5), we obtain

$$
\begin{aligned}
& {\left[\frac{(\hat{k}-1) C(\hat{s})}{2}+\hat{s} B(\hat{s})\right] \sum_{k=\hat{k}}^{\infty} p_{i k}(t) k \hat{s}^{k-2}} \\
& \quad \geq \sum_{j=0}^{\infty} p_{i j}^{\prime}(t) \hat{s}^{j}-\sum_{k=1}^{k-1}\left[\frac{(k-1) C(\hat{s})}{2}+\hat{s} B(\hat{s})\right] p_{i k}(t) k \hat{s}^{k-2} .
\end{aligned}
$$

Integrating the above inequality yields

$$
\begin{aligned}
& {\left[\frac{(\hat{k}-1) C(\hat{s})}{2}+\hat{s} B(\hat{s})\right] \sum_{k=\hat{k}}^{\infty}\left(\int_{0}^{\infty} p_{i k}(t) \mathrm{d} t\right) k \hat{s}^{k-2}} \\
& \quad \geq \lim _{t \rightarrow \infty} p_{i 0}(t)-\hat{s}^{i}-\sum_{k=1}^{k-1}\left[\frac{(k-1) C(\hat{s})}{2}+\hat{s} B(\hat{s})\right]\left(\int_{0}^{\infty} p_{i k}(t) \mathrm{d} t\right) k \hat{s}^{k-2} \\
& >-\infty,
\end{aligned}
$$

which implies $(2.3)$ since $\frac{1}{2}(\hat{k}-1) C(\hat{s})+\hat{s} B(\hat{s})<0$. The proof is complete.

\section{Uniqueness}

In order to discuss the regularity and uniqueness, we also need the following result.

Lemma 3.1. Suppose that $Q=\left(q_{i j} ; i, j \geq 0\right)$ is a conservative $q$-matrix and $\bar{k} \geq 1$ is an integer. Define a new matrix $Q^{*}=\left(q_{i j}^{*} ; i, j \geq 0\right)$ as

$$
q_{i j}^{*}= \begin{cases}q_{i j} & \text { if } i>\bar{k}, \\ 0 & \text { otherwise. }\end{cases}
$$

Then $Q^{*}$ is also a conservative $q$-matrix. Moreover, if $Q$ is regular then so is $Q^{*}$. 
Proof. We only need to prove the last conclusion. Suppose that $Q^{*}$ is not regular. Then the equation

$$
Q^{*} Y \geq \lambda Y
$$

has a nontrivial nonnegative and bounded solution for some $\lambda>0$, denoted by $Y=\left(y_{i} ; i \geq 0\right)$. It is easily seen that $y_{i}=0$ for $i \leq \bar{k}$. We claim that $Y=\left(y_{i} ; i \geq 0\right)$ is also a solution of

$$
Q Y \geq \lambda Y \text {. }
$$

Indeed, for $i \leq \bar{k}$,

$$
(Q Y)_{i}=\sum_{j=0}^{\infty} q_{i j} y_{j}=\sum_{j=\bar{k}+1}^{\infty} q_{i j} y_{j} \geq 0=\lambda y_{i},
$$

since $y_{i}=0$ for all $i \leq \bar{k}$. For $i>\bar{k}$,

$$
(Q Y)_{i}=\left(Q^{*} Y\right)_{i} \geq \lambda y_{i} .
$$

Therefore, $Q$ is not regular. The proof is complete.

In this section we consider regularity and uniqueness for IBCPs. For convenience, from now on, we will always assume that $C^{\prime}(1)+B^{\prime}(1)<+\infty$ for any given IBC $q$-matrix $Q$.

Theorem 3.1. Let $Q$ be an IBC q-matrix as defined in (1.1)-(1.2). Then $Q$ is regular if and only if $C^{\prime}(1) \leq 0$.

Proof. First assume that $C^{\prime}(1) \leq 0$. If we further assume that $B^{\prime}(1) \leq 0$ then both $C(s)$ and $B(s)$ are positive for all $s \in[0,1)$. It follows from (2.3) that

$$
\lambda \sum_{j=0}^{\infty} \phi_{i j}(\lambda) s^{j} \geq s^{i}, \quad s \in[0,1) .
$$

Letting $s \uparrow 1$ in (3.1) yields $\lambda \sum_{j=0}^{\infty} \phi_{i j}(\lambda)=1$, i.e. $Q$ is regular.

Now suppose that $C^{\prime}(1) \leq 0$ and $B^{\prime}(1)>0$. Since $B^{\prime}(1)<+\infty$ and $C^{\prime}(1) \leq 0$, we obtain, from (2.2),

$$
\lambda \sum_{j=0}^{\infty} \phi_{i j}(\lambda) s^{j}-s^{i} \geq B(s) \sum_{k=1}^{\infty} \phi_{i k}(\lambda) k s^{k-1}, \quad s \in[0,1) .
$$

If $Q$ is not regular then there exist an $i \geq 0$ and a $\lambda>0$ such that $\lambda \sum_{j=0}^{\infty} \phi_{i j}(\lambda)<1$. Hence, there exist a $\delta>0$ and an $\tilde{s} \in\left(\rho_{b}, 1\right)$ such that, for all $s \in[\tilde{s}, 1]$, we have

$$
s^{i}-\lambda \sum_{j=0}^{\infty} \phi_{i j}(\lambda) s^{j}>\delta .
$$

Note that $B(s)<0$ for all $\tilde{s} \in\left(\rho_{b}, 1\right)$; thus, by using (3.2) and (3.3), we obtain

$$
\sum_{k=1}^{\infty} \phi_{i k}(\lambda) k s^{k-1} \geq \frac{s^{i}-\lambda \sum_{j=0}^{\infty} \phi_{i j}(\lambda) s^{j}}{-B(s)} \geq \frac{\delta}{-B(s)}, \quad s \in[\tilde{s}, 1) .
$$

Therefore,

$$
\sum_{k=1}^{\infty} \phi_{i k}(\lambda)\left(1-\tilde{s}^{k}\right) \geq \int_{\tilde{s}}^{1} \frac{\delta}{-B(s)} \mathrm{d} s=+\infty
$$

which is a contradiction and, hence, $Q$ is regular. 
We now prove the converse. Suppose that $C^{\prime}(1)>0$. By a similar argument as in Chen et al. [6], we can find two constants $a^{*}$ and $b^{*}$ such that

$$
2 c_{0}+c_{1}<a^{*}<b^{*}<\sum_{j=1}^{\infty} j c_{j+2}
$$

and

$$
\sum_{j=1}^{\infty} c_{j+2} \sum_{k=1}^{j}\left(\frac{a^{*}}{b^{*}}\right)^{k-1}>b^{*}
$$

Now, we choose a $\varepsilon \in\left(0, b^{*}-a^{*}\right)$ and let $i_{0}=\left[2 b_{0} / \varepsilon\right]+1$, and then define a $q$-matrix $\tilde{Q}=\left(\tilde{q}_{i j} ; i, j \geq 0\right)$ as

$$
\tilde{q}_{i j}= \begin{cases}\left(\begin{array}{l}
i \\
2
\end{array}\right) c_{j-i+2}+i b_{j-i+1} & \text { if } i>i_{0}, j \geq i-2 \\
0 & \text { otherwise. }\end{cases}
$$

By Lemma 3.1 we only need to prove that $\tilde{Q}$ is not regular. For this purpose, define a (conservative) birth-death $q$-matrix $Q^{*}=\left(q_{i j}^{*} ; i, j \in \mathbb{Z}_{+}\right)$by

$$
q_{i j}^{*}= \begin{cases}\left(\begin{array}{l}
i \\
2
\end{array}\right) b^{*} & \text { if } i>i_{0}, j=i+1, i \geq 2, \\
\left(\begin{array}{l}
i \\
2
\end{array}\right)\left(a^{*}+\varepsilon\right) & \text { if } i>i_{0}, j=i-1, i \geq 2, \\
-\left(\begin{array}{l}
i \\
2
\end{array}\right)\left(b^{*}+a^{*}+\varepsilon\right) & \text { if } j=i>i_{0}, \\
0 & \text { otherwise. }\end{cases}
$$

Since $b^{*}>a^{*}+\varepsilon>0$ and $\sum_{i=2}^{\infty}\left(\begin{array}{l}i \\ 2\end{array}\right)^{-1}<+\infty$, it is easy to see that $Q^{*}$ is not regular. Hence, the equation

$$
\left(\lambda I-Q^{*}\right) u=0, \quad \lambda>0,
$$

has a nontrivial (nonnegative) bounded solution, denoted by $u^{*}=\left(u_{i} ; i \geq 0\right)$. Clearly, $u_{i}>0$ for all $i>i_{0}$. It is also easy to see that $u_{0}=\cdots=u_{i_{0}}=0$ and

$$
b^{*}\left(u_{i+1}-u_{i}\right)=\left(a^{*}+\varepsilon\right)\left(u_{i}-u_{i-1}\right)+\lambda u_{i}\left(\begin{array}{l}
i \\
2
\end{array}\right)^{-1}, \quad i>i_{0} .
$$

In particular, for $i=i_{0}+1$, we have $b^{*}\left(u_{i_{0}+2}-u_{i_{0}+1}\right)=\left(a^{*}+\varepsilon+\lambda\right) u_{i_{0}+1}(>0)$, which implies that $\left(u_{i} ; i>i_{0}\right)$ is strictly increasing in $i$. From (3.5), it is easily seen that, for all $k \geq 1$ and $i>i_{0}$,

$$
u_{i+k}-u_{i+k-1} \geq\left(\frac{a^{*}+\varepsilon}{b^{*}}\right)^{k-1}\left(u_{i+1}-u_{i}\right)>\left(\frac{a^{*}}{b^{*}}\right)^{k-1}\left(u_{i+1}-u_{i}\right)
$$

and

$$
u_{i-1}-u_{i-2} \leq \frac{b^{*}}{a^{*}+\varepsilon}\left(u_{i}-u_{i-1}\right)<\frac{b^{*}}{a^{*}}\left(u_{i}-u_{i-1}\right) .
$$


Now, for $i>i_{0}$, we have

$$
\begin{aligned}
(\tilde{Q} u)_{i}= & \left(\begin{array}{l}
i \\
2
\end{array}\right)\left(c_{0}\left(u_{i-2}-u_{i}\right)+c_{1}\left(u_{i-1}-u_{i}\right)+\sum_{j=i+1}^{\infty} c_{j-i+2}\left(u_{j}-u_{i}\right)\right) \\
& +i\left[b_{0}\left(u_{i-1}-u_{i}\right)+\sum_{j=i+1}^{\infty} b_{j-i+1}\left(u_{j}-u_{i}\right)\right] \\
= & \left(\begin{array}{l}
i \\
2
\end{array}\right)\left(-I_{d}+I_{b}\right)+i\left(-J_{d}+J_{b}\right),
\end{aligned}
$$

where $I_{d}, I_{b}, J_{d}$, and $J_{b}$ are self-explanatory by the above.

Now, by (3.5) and (3.6), we obtain

$$
I_{b} \geq \sum_{j=1}^{\infty} c_{j+2} \sum_{k=1}^{j}\left(\frac{a^{*}}{b^{*}}\right)^{k-1}\left(u_{i+1}-u_{i}\right)>b^{*}\left(u_{i+1}-u_{i}\right)
$$

and

$$
J_{b} \geq \sum_{j=1}^{\infty} b_{j+1} \sum_{k=1}^{j}\left(\frac{a^{*}}{b^{*}}\right)^{k-1}\left(u_{i+1}-u_{i}\right)=\tilde{b}\left(u_{i+1}-u_{i}\right),
$$

where $\tilde{b}=\sum_{j=1}^{\infty} b_{j+1} \sum_{k=1}^{j}\left(a^{*} / b^{*}\right)^{k-1}$.

Similarly, by (3.7) we have

$$
I_{d} \leq\left(c_{0} \frac{b^{*}}{a^{*}}+\left(c_{0}+c_{1}\right)\right)\left(u_{i}-u_{i-1}\right)<a^{*}\left(u_{i}-u_{i-1}\right)
$$

and $J_{d}=b_{0}\left(u_{i}-u_{i-1}\right)$. Therefore,

$$
\left(\begin{array}{l}
i \\
2
\end{array}\right) I_{b}+i J_{b} \geq\left(\begin{array}{l}
i \\
2
\end{array}\right) b^{*}\left(u_{i+1}-u_{i}\right)+i \tilde{b}\left(u_{i+1}-u_{i}\right)
$$

and

$$
\begin{aligned}
\left(\begin{array}{l}
i \\
2
\end{array}\right) I_{d}+i J_{d} & \leq\left(\begin{array}{l}
i \\
2
\end{array}\right) a^{*}\left(u_{i+1}-u_{i}\right)+i b_{0}\left(u_{i+1}-u_{i}\right) \\
& =\left(\begin{array}{l}
i \\
2
\end{array}\right)\left(a^{*}+\varepsilon\right)\left(u_{i}-u_{i-1}\right)-\left[\left(\begin{array}{l}
i \\
2
\end{array}\right) \varepsilon-i b_{0}\right]\left(u_{i}-u_{i-1}\right) .
\end{aligned}
$$

Therefore, $u^{*}=\left(u_{i} ; i \geq 0\right)$ satisfies

$$
\tilde{Q} u^{*} \geq \lambda u^{*}
$$

Indeed, (3.9) is obviously true for $i \leq i_{0}$. For $i>i_{0}$, using (3.7)-(3.8) and (3.4), we can easily obtain

$$
(\tilde{Q} u)_{i} \geq \lambda u_{i}+\left[\left(\begin{array}{l}
i \\
2
\end{array}\right) \varepsilon-i b_{0}\right]\left(u_{i}-u_{i-1}\right) \geq \lambda u_{i} .
$$

Thus, $\tilde{Q}$ is not regular and, hence, by Lemma 3.1, $Q$ is not regular. The proof is complete. 
Remark 3.1. Theorem 3.1 provides a regularity criterion under the assumption (see the beginning of this section) that $C^{\prime}(1)+B^{\prime}(1)<+\infty$. However, by checking the proof carefully, one will find that the conclusion still holds if $C^{\prime}(1)=+\infty$, provided that $B^{\prime}(1)<+\infty$. Even if this latter condition is removed, $Q$ may still be regular. For example, we can prove that if $C^{\prime}(1) \leq 0, B^{\prime}(1)>0$, and

$$
\int_{\gamma}^{1} \frac{1}{C(s)} \exp \left\{\int_{0}^{s} \frac{2 B(x)}{C(x)} \mathrm{d} x\right\} \mathrm{d} s=+\infty
$$

for some (or, equivalently, for all) $\gamma \in\left(\rho_{b}, 1\right)$, then $Q$ is regular. Indeed, suppose that $Q$ is not regular. Then there exist $\varepsilon$ and $\gamma \in\left(\rho_{b}, 1\right)$ such that $s^{i}-\lambda \Phi(s)>\varepsilon>0$ for all $s \in(\gamma, 1)$. Therefore, by (2.4) we have

$$
\Phi^{\prime}(s) \exp \left\{\int_{\gamma}^{s} \frac{2 B(x)}{C(x)} \mathrm{d} x\right\}-\Phi^{\prime}(\gamma) \leq-2 \varepsilon \int_{\gamma}^{s} \frac{1}{C(y)} \exp \left\{\int_{\gamma}^{y} \frac{2 B(x)}{C(x)} \mathrm{d} x\right\} \mathrm{d} y .
$$

Letting $s \rightarrow 1$ in the above inequality yields $\lim _{s \rightarrow 1} \Phi^{\prime}(s) \exp \left\{\int_{\gamma}^{s} 2 B(x) \mathrm{d} x / C(x)\right\}=-\infty$, which is a contradiction. Hence, $Q$ is regular.

We now consider the uniqueness problem of $Q$-functions which satisfy the Kolmogorov forward equation.

Theorem 3.2. There always exists exactly one $Q$-function that satisfies the Kolmogorov forward equation. That is, there always exists only one IBCP which is the Feller minimal process for any given $Q$.

Proof. By Theorem 3.1 we only need to consider the case that $C^{\prime}(1)>0$. By Theorem 2.2.8 of [1], we only need to show that the equation

$$
\lambda Y=Y Q, \quad Y \geq 0, Y \cdot \mathbf{1}<+\infty,
$$

has no nontrivial solutions for some (and then for all) $\lambda>0$, where $Y \cdot \mathbf{1}$ denotes the inner product of $Y$ and the vector 1 whose components are all 1 .

Suppose that $Y=\left(y_{k} ; k \geq 0\right)$ is a nontrivial solution of (3.10) with $\lambda=1$. Then $y_{0}>0$ and (3.10) can be written as

$$
Y(s)=\frac{C(s)}{2} Y^{\prime \prime}(s)+B(s) Y^{\prime}(s), \quad|s|<1,
$$

where $Y(s)=\sum_{k=0}^{\infty} y_{k} s^{k}$.

First consider the case that $C^{\prime}(1)>0$. If $B^{\prime}(1)>0$, we have both $C(s)<0$ and $B(s)<0$ for all $s \in\left(\rho_{c} \vee \rho_{b}, 1\right)$, and, hence, the right-hand side of (3.11) is negative. However, the left-hand side is positive, which is a contradiction. If $B^{\prime}(1) \leq 0$ then

$$
Y(s) \leq B(s) Y^{\prime}(s), \quad s \in\left(\rho_{c}, 1\right) .
$$

Hence,

$$
\ln Y(1)-\ln Y\left(\rho_{c}\right) \geq \int_{\rho_{c}}^{1} \frac{\mathrm{d} s}{B(s)}=+\infty,
$$

which contradicts $0<Y(1)<\infty$. 


\section{Extinction probability: regular case}

Let $\{X(t) ; t \geq 0\}$ be the unique IBCP with a given IBC $q$-matrix $Q$ as defined in (1.1)-(1.2), and let $P(t)=\left(p_{i j}(t) ; i, j \in \mathbb{Z}_{+}\right)$be the unique $Q$-function. Let

$$
\tau_{0}=\inf \{t>0 ; X(t)=0\}
$$

and

$$
a_{i}=\mathrm{P}\left(\tau_{0}<\infty \mid X(0)=i\right), \quad i \geq 1,
$$

be the extinction time and extinction probability, respectively.

We will consider the absorbing behavior of an IBCP in two different cases, regular and irregular, in this and the next section, respectively. As a preparation, we first provide two lemmas which hold for both cases.

Define

$$
G_{i}(s)=\sum_{k=1}^{\infty}\left(\int_{0}^{\infty} p_{i k}(t) \mathrm{d} t\right) s^{k}, \quad i \geq 1 .
$$

Then by (2.3) we know that $G_{i}(s)$ is well defined for all $|s|<1$.

In addition, define

$$
H(y)=\int_{0}^{y} \frac{B(x)}{C(x)} \mathrm{d} x, \quad y \in\left(\zeta_{c}, \rho_{c}\right),
$$

where the integral should be taken along the inverse direction if $y<0$. By Lemma 2.1 we know that $H(y)$ is finite for all $y \in\left(\zeta_{c}, \rho_{c}\right)$. Also, let

$$
A(y)=\exp \{2 H(y)\}, \quad y \in\left(\zeta_{c}, \rho_{c}\right) .
$$

It is obvious that $H(0)=0$ and $H(y)<0$ if $y \in\left(\zeta_{c}, 0\right)$, and, hence, $A(0)=1$ and $A(y)<1$ if $y \in\left(\zeta_{c}, 0\right)$. It is also clear that $A(y) \rightarrow 0$ if and only if $H(y) \rightarrow-\infty$. The following simple lemma provides further information about the two functions $H(y)$ and $A(y)$ which will be useful in our later analysis. It is easy to verify the correctness of Lemma 4.1 below and, thus, we will only prove Lemma 4.2 below.

Lemma 4.1. (i) $\lim _{y \rightarrow \zeta_{c}^{+}} H(y)=-\infty$ and $\lim _{y \rightarrow \zeta_{c}^{+}} A(y)=0$. Moreover, we have $A(y) \sim$ $K\left(y-\zeta_{c}\right)$ when $y \rightarrow \zeta_{c}^{+}$, where $0<K<+\infty$ and, in fact, $K=2 B\left(\zeta_{c}\right) / C^{\prime}\left(\zeta_{c}\right)$.

(ii) Suppose that $0<C^{\prime}(1) \leq+\infty$ and, thus, $\rho_{c}<1$. If $\rho_{b}=\rho_{c}<1$ then $0 \leq H\left(\rho_{c}\right)<$ $+\infty$. If $\rho_{b}<\rho_{c}<1$ then $\lim _{y \rightarrow \rho_{c}^{-}} H(y)=-\infty$ and, hence, $\lim _{y \rightarrow \rho_{c}^{-}} A(y)=0$. Moreover, $A(y) \sim K\left(\rho_{c}-y\right)$ when $y \rightarrow \rho_{c}^{-}$, where $0<K<+\infty$ and, in fact, $K=2 B\left(\rho_{c}\right) / C^{\prime}\left(\rho_{c}\right)$. If $\rho_{c}<\rho_{b} \leq 1$ then $\lim _{y \rightarrow \rho_{c}^{-}} H(y)=+\infty$.

Lemma 4.2. (i) For any $i \geq 1$ and $|s|<1$,

$$
\frac{C(s)}{2} G_{i}^{\prime \prime}(s)+B(s) G_{i}^{\prime}(s)=a_{i}-s^{i} .
$$

Moreover, for $|s|<\rho_{c}$, we have

$$
G_{i}^{\prime}(s) A(s)-G_{i}^{\prime}(0)=\int_{0}^{s} \frac{2\left(a_{i}-y^{i}\right)}{C(y)} A(y) \mathrm{d} y .
$$

(ii) For any $i \geq 1$,

$$
\lim _{s \rightarrow \zeta_{c}} G_{i}^{\prime}(s) A(s)=0
$$


Proof. Integrating (2.1) with respect to $t \in[0, \infty)$ immediately yields (4.3) and then (4.4) immediately follows.

We now turn to the proof of (4.5). If $-1<\zeta_{c}<0$ then the proof is easy. Indeed, since for this case we have $\left|G_{i}^{\prime}\left(\zeta_{c}\right)\right|<\infty$, then by Lemma 4.1 we know that $\lim _{s \rightarrow \zeta_{c}} A(s)=0$, and (4.5) follows.

If $\zeta_{c}=-1$, the proof is little bit lengthy. Recall Lemma 4.1. We know that if $\zeta_{c}=-1$ then $C(-x)=C(x)$ for any $x \in[0,1]$. Note that, for any $x \in(0,1)$,

$$
B(-x)+B(x)=2 \sum_{k=0}^{\infty} b_{2 k} x^{2 k}>2 b_{0}, \quad \text { i.e. }-B(-x)<B(x)-2 b_{0} .
$$

Therefore, for any $s \in[0,1)$,

$$
\begin{aligned}
A(-s) & =\exp \left\{\int_{0}^{-s} \frac{2 B(x)}{C(x)} \mathrm{d} x\right\} \\
& =\exp \left\{-\int_{0}^{s} \frac{2 B(-x)}{C(x)} \mathrm{d} x\right\} \\
& \leq \exp \left\{\int_{0}^{s} \frac{2 B(x)-4 b_{0}}{C(x)} \mathrm{d} x\right\} \\
& =A(s) \exp \left\{-4 b_{0} \int_{0}^{s} \frac{\mathrm{d} x}{C(x)}\right\} .
\end{aligned}
$$

It follows from (4.4) that, for $s \in[0,1)$,

$$
\begin{aligned}
\left|G_{i}^{\prime}(-s)\right| A(-s) \leq & G_{i}^{\prime}(s) A(s) \exp \left\{-4 b_{0} \int_{0}^{s} \frac{\mathrm{d} x}{C(x)}\right\} \\
\leq & G_{i}^{\prime}(0) \exp \left\{-4 b_{0} \int_{0}^{s} \frac{\mathrm{d} x}{C(x)}\right\} \\
& +\left(2 \int_{0}^{s} \frac{A(y)}{C(y)} \mathrm{d} y\right) \exp \left\{-4 b_{0} \int_{0}^{s} \frac{\mathrm{d} x}{C(x)}\right\} \\
\leq & G_{i}^{\prime}(0) \exp \left\{-4 b_{0} \int_{0}^{s} \frac{\mathrm{d} x}{C(x)}\right\} \\
& +2\left(\int_{0}^{s} \frac{\exp \left\{2 b_{0} \int_{0}^{y} \mathrm{~d} x / C(x)\right\}}{C(y)} \mathrm{d} y\right) \exp \left\{-4 b_{0} \int_{0}^{s} \frac{\mathrm{d} x}{C(x)}\right\} \\
\leq & G_{i}^{\prime}(0) \exp \left\{-4 b_{0} \int_{0}^{s} \frac{\mathrm{d} x}{C(x)}\right\} \\
& +2\left(\int_{0}^{s} \frac{\exp \left\{-b_{0} \int_{0}^{y} \mathrm{~d} x / C(x)\right\}}{C(y)} \mathrm{d} y\right) \exp \left\{-b_{0} \int_{0}^{s} \frac{\mathrm{d} x}{C(x)}\right\} \\
\leq & G_{i}^{\prime}(0) \exp \left\{-4 b_{0} \int_{0}^{s} \frac{\mathrm{d} x}{C(x)}\right\}+\frac{2}{b_{0}} \exp \left\{-b_{0} \int_{0}^{s} \frac{\mathrm{d} x}{C(x)}\right\} .
\end{aligned}
$$

Therefore,

$$
\lim _{s \rightarrow \zeta_{c}}\left|G_{i}^{\prime}(s)\right| A(s)=\lim _{s \rightarrow 1}\left|G_{i}^{\prime}(-s)\right| A(-s)=0,
$$

and, thus, (4.5) is proved. 
We are now ready to consider the case that $Q$ is regular. The main conclusions regarding the extinction probability for this regular case will be provided below. The irregular, i.e. explosive, case will be discussed in the next section. First recall that, by Theorem 3.1, an IBCP $q$-matrix $Q$ is regular if and only if $C^{\prime}(1) \leq 0$.

Theorem 4.1. Suppose that $C^{\prime}(1) \leq 0$ and $B^{\prime}(1) \leq 0$. Then $a_{i}=1(i \geq 1)$.

Proof. Since $C^{\prime}(1) \leq 0$ and $B^{\prime}(1) \leq 0$, we know by Lemma 2.1 that $C(s)>0$ and $B(s)>0$ for all $s \in[0,1)$. It follows from (4.3) that $a_{i}-s^{i} \geq 0$ for all $s \in[0,1)$ and, thus, by letting $s \rightarrow 1$ we obtain $a_{i} \geq 1$. But $a_{i} \leq 1$ is always true and, thus, $a_{i}=1$.

Theorem 4.2. Suppose that $C^{\prime}(1) \leq 0$ and $0<B^{\prime}(1) \leq+\infty$. Then $a_{i}=1(i \geq 1)$ if and only if $J=+\infty$, where

$$
J=\int_{\zeta_{c}}^{1} \frac{A(y)}{C(y)} \mathrm{d} y
$$

and $A(y)$ is as defined in (4.2). Moreover, if $J<+\infty$ then

$$
a_{i}=J^{-1} \int_{\zeta_{c}}^{1} \frac{y^{i} A(y)}{C(y)} \mathrm{d} y, \quad i \geq 1 .
$$

Proof. Suppose that $J=+\infty$. By the communicating property of the positive states, we know that either $a_{i}=1$ for all $i \geq 1$ or $a_{i}<1$ for all $i \geq 1$. Now, assume that $a_{1}<1$. We rewrite (4.4) as

$$
G_{1}^{\prime}(s) A(s)-G_{1}^{\prime}(0)=2 \int_{0}^{a_{1}} \frac{a_{1}-y}{C(y)} A(y) \mathrm{d} y+2 \int_{a_{1}}^{s} \frac{a_{1}-y}{C(y)} A(y) \mathrm{d} y .
$$

Let $s \rightarrow 1$ in equality (4.8). Then the first term on the right-hand side of (4.8) is obviously a finite constant and the last term tends to $-\infty$ since $J=+\infty$. This latter fact could be easily seen by applying the integral mean-values theorem. However, the left-hand side is either finite or $+\infty$. This contradiction yields the result $a_{i}=1$ for all $i \geq 1$.

Now suppose that $J<+\infty$. By (4.4) and (4.5),

$$
-G_{i}^{\prime}(0)=-2 \int_{\zeta_{c}}^{0} \frac{a_{i}-y^{i}}{C(y)} A(y) \mathrm{d} y .
$$

It follows from (4.9) and (4.4) that, for $i \geq 1$ and $s \in\left(\zeta_{c}, 1\right)$,

$$
G_{i}^{\prime}(s) A(s)=\int_{\zeta_{c}}^{s} \frac{2\left(a_{i}-y^{i}\right)}{C(y)} A(y) \mathrm{d} y .
$$

Define

$$
x_{i}=J^{-1} \int_{\zeta_{c}}^{1} \frac{y^{i} A(y)}{C(y)} \mathrm{d} y, \quad i \geq 1 .
$$

Then, by (4.10), $a_{i} \geq x_{i}(i \geq 1)$. On the other hand, it can be shown that $\left(x_{i} ; i \geq 1\right)$ is a solution of the equation

$$
\sum_{k=1}^{\infty} q_{i k} x_{k}+q_{i 0}=0, \quad 0 \leq x_{i} \leq 1, i \geq 1 .
$$


Indeed, for $i \geq 1$,

$$
\begin{aligned}
\sum_{k=1}^{\infty} q_{i k} x_{k}+q_{i 0} & =\frac{1}{2 J}\left[i(i-1) \int_{\zeta_{c}}^{1} y^{i-2} A(y) \mathrm{d} y+i \int_{\zeta_{c}}^{1} y^{i-1} A^{\prime}(y) \mathrm{d} y\right] \\
& =\frac{i}{2 J}\left[A(1)-\zeta^{i-1} A\left(\zeta_{c}\right)\right] \\
& =0
\end{aligned}
$$

where the last step follows from Lemma 4.1 and the fact that the assumption $J<\infty$ implies that $A(1)=0$. Therefore, by Lemma 4.46 of [7] (or [12]), we know that $a_{i} \leq x_{i}(i \geq 1)$. Hence, (4.7) is proved. The proof is complete.

Remark 4.1. It is easy to see that $J<+\infty$ if and only if $J_{0}=\int_{0}^{1}(A(y) / C(y)) \mathrm{d} y<+\infty$, where $J$ is given in (4.6). Note that $J_{0}$ is much easier to check since we do not need to know the value of $\zeta_{c}$, which may be quite difficult to obtain.

In practice, it may be difficult to check whether $J$ or even $J_{0}$ is finite or not. Fortunately, in most practical cases, we do not need to check either of them. The following corollaries give some convenient sufficient conditions.

Corollary 4.1. Suppose that $C^{\prime}(1)<0$ and $0<B^{\prime}(1)<+\infty$. Then $a_{i}=1(i \geq 1)$.

Proof. Note that $A(1)=\exp \left\{\int_{0}^{1}(2 B(x) / C(x)) \mathrm{d} x\right\}>0$ and, thus, $J=+\infty$; the required conclusion immediately follows from Theorem 4.2.

If $C^{\prime}(1)<0$ but $B^{\prime}(1)=+\infty$, then to guarantee that $a_{i}=1$, we need some further conditions. The next corollary provides such a simple sufficient condition which could be easily applied.

Corollary 4.2. Suppose that $C^{\prime}(1)<0$ and $B^{\prime}(1)=+\infty$. Furthermore, assume that there exist a $\delta>0$ and some positive constant $k$ such that

$$
B(s) \sim(-k)(1-s)^{\delta} \quad \text { as } s \uparrow 1 .
$$

Then, for all $i \geq 1$, we have $a_{i}=1$.

Proof. Just note that we have $B(s) / C(s) \sim(-1) k_{1} /(1-s)^{1-\delta}, s \uparrow 1$, where $0<k_{1}<\infty$ under the condition of this corollary. Hence, $\int_{0}^{1}(B(x) / C(x)) \mathrm{d} x$ is convergent, guaranteeing that $A(y)$ is bounded on $[0,1]$, which, in turn, implies that $J_{0}=+\infty$.

Of course, the only possible value of $\delta>0$ in Corollary 4.2 is $\delta<1$ since $B^{\prime}(1)=+\infty$ implies that $\lim _{s \uparrow 1}(-1) B(s) /(1-s)=+\infty$. Now we consider the case that $C^{\prime}(1)=0$.

Corollary 4.3. Suppose that $C^{\prime}(1)=0$ and $0<B^{\prime}(1)<+\infty$, together with the additional assumptions that both $C^{\prime \prime}(1)$ and $B^{\prime \prime}(1)$ are finite. If $C^{\prime \prime}(1) \geq 4 B^{\prime}(1)$ then $J=+\infty$; thus, $a_{i}=1(i \geq 1)$. If $C^{\prime \prime}(1)<4 B^{\prime}(1)$ then $J<+\infty$; thus, $a_{i}<1(i \geq 1)$ and then $a_{i}$ is given by (4.7).

Proof. Suppose that $C^{\prime}(1)=0$. Define $\gamma=4 B^{\prime}(1) / C^{\prime \prime}(1)$. First note that we have the obvious fact that $C^{\prime \prime}(1)>0$ and, thus, $0<\gamma<+\infty$ under the condition of this corollary. Secondly, let $g(x)=2(1-x) B(x) / C(x)$. Then $g(x)$ is an analytic function on the disk $\left\{z ;|z|<\left|\zeta_{c}\right|\right\}$, where $\zeta_{c}$ is the unique negative zero of $C(x)$ (also note that $C(x)$ has no zero 
in $[0,1))$ and, thus, could be expanded as a power series of $x$ in the internal $[0,1)$ with the form $g(x)=\sum_{k=0}^{\infty} g_{k} x^{k}$, say. Then the $H(y)$ defined in (4.1) for $y \geq 0$ could be expressed as

$$
H(y)=\frac{1}{2} \sum_{k=0}^{\infty} g_{k} \int_{0}^{y} \frac{x^{k}}{1-x} \mathrm{~d} x,
$$

which could be rewritten as, by simply letting $x=1-(1-x)$,

$$
H(y)=-\frac{\ln (1-y)}{2} \sum_{k=0}^{\infty} g_{k}+H_{1}(y),
$$

where $H_{1}(y)=\frac{1}{2} \sum_{k=1}^{\infty} g_{k} \int_{0}^{y} \sum_{m=1}^{k}(-1)^{m}(1-x)^{m-1} \mathrm{~d} x$ (a further simple form for $H_{1}(y)$ is of course available but not necessary here) is a bounded function of $y$ on $[0,1]$.

Now noting that

$$
\sum_{k=0}^{\infty} g_{k}=\lim _{x \uparrow 1} \frac{2(1-x) B(x)}{C(x)}=\frac{(-4) B^{\prime}(1)}{C^{\prime \prime}(1)}=-\gamma
$$

(here we have used the fact that $\left.B^{\prime \prime}(1)<+\infty\right)$, the $A(y)$ defined in (4.2) could therefore be written as

$$
A(y)=A_{1}(y)(1-y)^{\gamma},
$$

where $A_{1}(y)$ is a bounded function of $y \in[0,1]$.

It then follows that $J_{0}$ (and, thus, $J$ ) is finite if and only if the integral $\int_{0}^{1}(1-y)^{\gamma-2} \mathrm{~d} y$ is convergent, or, equivalently, if and only if $4 B^{\prime}(1)>C^{\prime \prime}(1)$. The proof is now complete.

Corollary 4.4. Suppose that $C^{\prime}(1)=0,0<C^{\prime \prime}(1)<4 B^{\prime}(1)<+\infty$, and $B^{\prime \prime}(1)$ is finite. Then $a_{i} \rightarrow 0$ as $i \rightarrow \infty$ and

$$
\sum_{i=1}^{\infty} a_{i}=J^{-1} \int_{\zeta_{c}}^{1} \frac{y A(y)}{(1-y) C(y)} \mathrm{d} y,
$$

which is finite if and only if $\int_{0}^{1}(y A(y) /(1-y) C(y)) \mathrm{d} y<\infty$. More exactly, we have $a_{i} \sim$ $k / i^{\gamma-1}$ as $i \rightarrow+\infty$, where $k$ is a constant and $\gamma=4 B^{\prime}(1) / C^{\prime \prime}(1)>1$. Moreover, $\sum_{i=1}^{\infty} a_{i}$ is finite if and only if $2 B^{\prime}(1)>C^{\prime \prime}(1)$.

Proof. By Corollary 4.3 we know that $J<+\infty$ and that, for all $i \geq 1$, we have $a_{i}<1$ and, thus, (4.11) follows from (4.7) directly. By a similar approach to that used to prove Corollary 4.3, we could easily show that $a_{i} \sim k_{1} B(i+1, \gamma-1)$ as $i \rightarrow+\infty$, where $k_{1}$ is a positive constant, $\gamma=4 B^{\prime}(1) / C^{\prime \prime}(1)>1$, and $B(a, b)$ is the beta function. Now using the well-known result that $\lim _{z \rightarrow+\infty} \Gamma(z+\alpha) z^{-\alpha} / \Gamma(z)=1$ (which is true even for a complex number $\alpha$ with positive real part), where $\Gamma(x)$ is the gamma function, we immediately obtained the result that there exists some positive constant $k$ such that $a_{i} \sim k / i^{\gamma-1}$ as $i \rightarrow \infty$. Now $a_{i} \rightarrow 0$ also immediately follows.

Furthermore, again similarly as in the proof of Corollary 4.3, we could show that $\sum_{i=1}^{\infty} a_{i}$ is finite if and only if the integral $\int_{0}^{1}(1-y)^{\gamma-3} \mathrm{~d} y$ is convergent, or, equivalently, if and only if $2 B^{\prime}(1)>C^{\prime \prime}(1)$. This completes the proof.

Finally, we point out the following easy corollary. If the process takes the initial distribution $\Pi=\left(\pi_{i} ; i \geq 0\right)$ rather than a single-point distribution, then the extinction probability $a_{i}$ will be given as below, where we exclude the trivial case $\pi_{0}=1$. 
Corollary 4.5. Suppose that $C^{\prime}(1) \leq 0$ and the process starts with the initial distribution $\Pi=\left(\pi_{i} ; i \geq 0\right)$. Then the extinction probability $a_{\Pi}$ is as follows.

(i) If $B^{\prime}(1) \leq 0$ then $a_{\Pi}=1$.

(ii) If $0<B^{\prime}(1) \leq+\infty$ and $J_{0}=\int_{0}^{1}(A(y) / C(y)) \mathrm{d} y=+\infty$, then $a_{\Pi}=1$.

(iii) If $0<B^{\prime}(1) \leq+\infty$ and $J_{0}=\int_{0}^{1}(A(y) / C(y)) \mathrm{d} y<+\infty$, then

$$
a_{\Pi}=J^{-1} \int_{\zeta_{c}}^{1} \frac{A(y)}{\Pi(y) C(y)} \mathrm{d} y,
$$

where $\Pi(y)=\sum_{k=0}^{\infty} \pi_{k} y^{k}$.

In the final part of this section, we consider the mean extinction time for the regular case.

Theorem 4.3. Suppose that $Q$ is as given in (1.1)-(1.2) and that $P(t)=\left(p_{i j}(t) ; i, j \geq 0\right)$ is the Feller minimal $Q$-function. If $C^{\prime}(1) \leq 0$ and $J=+\infty$, then $\mathrm{E}_{i}\left[\tau_{0}\right]<\infty(i \geq 1)$ if and only if

$$
\int_{0}^{1}\left[\frac{1}{A(s)} \int_{0}^{s} \frac{(1-y) A(y)}{C(y)} \mathrm{d} y\right] \mathrm{d} s<\infty
$$

and $\mathrm{E}_{i}\left[\tau_{0}\right]$ is given by

$$
\mathrm{E}_{i}\left[\tau_{0}\right]=\int_{0}^{1}\left[\frac{1}{A(s)} \int_{\zeta_{c}}^{s} \frac{2\left(1-y^{i}\right) A(y)}{C(y)} \mathrm{d} y\right] \mathrm{d} s, \quad i \geq 1,
$$

where $\mathrm{E}_{i}$ is the conditional expectation when the process starts at $i \geq 1$.

Proof. By (4.4) and noting that $\zeta_{c}>-1$, we obtain

$$
G_{i}^{\prime}(s) A(s)-G_{i}^{\prime}(0)=\int_{0}^{s} \frac{2\left(1-y^{i}\right)}{C(y)} A(y) \mathrm{d} y
$$

and

$$
G_{i}^{\prime}(0)=\int_{\zeta_{c}}^{0} \frac{2\left(1-y^{i}\right)}{C(y)} A(y) \mathrm{d} y .
$$

Integrating (4.14) and noting that $G_{i}(0)=0$ yield

$$
G_{i}(s)=G_{i}^{\prime}(0) \int_{0}^{s} \frac{\mathrm{d} u}{A(u)}+\int_{0}^{s}\left[\frac{1}{A(u)} \int_{0}^{u} \frac{2\left(1-y^{i}\right)}{C(y)} A(y) \mathrm{d} y\right] \mathrm{d} u .
$$

By substituting (4.15) into the above equality we immediately obtain

$$
G_{i}(s)=\int_{0}^{s}\left[\frac{1}{A(u)} \int_{\zeta_{c}}^{u} \frac{2\left(1-y^{i}\right)}{C(y)} A(y) \mathrm{d} y\right] \mathrm{d} u .
$$

Letting $s \rightarrow 1$ in (4.16) yields

$$
G_{i}(1)=\int_{0}^{1}\left[\frac{1}{A(u)} \int_{\zeta_{c}}^{u} \frac{2\left(1-y^{i}\right)}{C(y)} A(y) \mathrm{d} y\right] \mathrm{d} u .
$$

By noting that $G_{i}(1)=\sum_{j=1}^{\infty} \int_{0}^{\infty} p_{i j}(t) \mathrm{d} t=\int_{0}^{\infty}\left(1-p_{i 0}(t)\right) \mathrm{d} t$ we immediately see that (4.13) follows from (4.17) directly. It is also obvious that (4.13) is finite if and only if (4.12) is true. 
If $C^{\prime}(1) \leq 0$ and $J<+\infty$, then, trivially, $\mathrm{E}_{i}\left[\tau_{0}\right]=+\infty$ due to the fact that $a_{i}<1$. To counteract this, we consider the informative conditional mean extinction time. Similarly as above, we may obtain the following conclusion.

Theorem 4.4. Suppose that $Q$ is an IBC q-matrix as in (1.1)-(1.2) and that $P(t)=\left(p_{i j}(t)\right.$; $i, j \geq 0)$ is the unique IBCP, i.e. the Feller minimal $Q$-function. If $C^{\prime}(1) \leq 0$ and $J<+\infty$, then

$$
a_{i} \mathrm{E}_{i}\left[\tau_{0} \mid \tau_{0}<\infty\right]=\int_{0}^{1}\left[\frac{1}{A(s)} \int_{\zeta_{c}}^{s} \frac{2\left(a_{i}-y^{i}\right) A(y)}{C(y)} \mathrm{d} y\right] \mathrm{d} s, \quad i \geq 1 .
$$

\section{Extinction probability: irregular case}

In the previous section we considered the situation that $Q$ is regular and satisfactory results regarding extinction properties were obtained. We now consider the case when $Q$ is irregular, or explosive. That is, we now consider the case that $0<C^{\prime}(1)<+\infty$ and, thus, $\rho_{c}<1$. Note that in this case the unique IBCP is still the Feller minimal $Q$-process and, thus, dishonest. We will see that this irregular case is much more subtle and it is necessary to further divide it into a few subcategories.

Definition 5.1. An irregular IBC $q$-matrix $Q$ is called superexplosive, critical explosive, or subexplosive if $\rho_{b}<\rho_{c}, \rho_{b}=\rho_{c}$, or $\rho_{b}>\rho_{c}$, respectively, where $\rho_{c}<1$ and $\rho_{b} \leq 1$ are the smallest nonnegative zeros of $C(s)$ and $B(s)$, respectively.

As far as extinction properties are concerned, the critical explosive case is the simplest case. Indeed, we have the following simple conclusion.

Theorem 5.1. If $Q$ is critical explosive, i.e. $\rho_{c}=\rho_{b}<1$, then $a_{i}=\rho_{c}^{i}(i \geq 1)$.

Proof. Note that $\rho_{c}<1$ is the common zero of $B(s)$ and $C(s)$, and both $G_{i}^{\prime}\left(\rho_{c}\right)$ and $G_{i}^{\prime \prime}\left(\rho_{c}\right)$ are finite; thus, by letting $s=\rho_{c}$ in (4.3) we immediately obtain the conclusion.

Under the condition that the corresponding $B(s)$ and $C(s)$ are polynomials of degrees 2 and 3, respectively, Theorem 5.1 was proved in [10]. One can see that, by our Theorem 5.1, this conclusion holds true for all situations. For more details, see Example 6.1 below.

For this critical explosive case, Kalinkin [10] did not consider the conditional mean extinction time. Here we would like to provide such conclusions for the general case.

Theorem 5.2. If $Q$ is critical explosive then the mean conditional extinction time $\mathrm{E}_{i}\left[\tau_{0} \mid \tau_{0}<\right.$ $\infty]$ is given by

$$
\mathrm{E}_{i}\left[\tau_{0} \mid \tau_{0}<\infty\right]=\rho_{c}^{-i} \int_{0}^{\rho_{c}}\left[\frac{2}{A(s)} \int_{\zeta_{c}}^{s}\left(1-\left(\frac{y}{\rho_{c}}\right)^{i}\right) \frac{A(y)}{C(y)} \mathrm{d} y\right] \mathrm{d} s,
$$

where $A(s)$ is defined in (4.2) and its domain is $\left(\zeta_{c}, 1\right)$.

Proof. First note that $\rho_{c}<1$ is the unique common simple zero of $B(s)$ and $C(s)$ on $[0,1)$, and, thus, $H(s)$ and $A(s)$ given in (4.1) and (4.2) are all well defined on [0,1). Hence, by solving

$$
\frac{C(s)}{2} G_{i}^{\prime \prime}(s)+B(s) G_{i}^{\prime}(s)=\rho_{c}^{i}-s^{i},
$$

we can prove this theorem by an argument similar to that used to prove Theorem 4.3. 
We now turn to the superexplosive case. Interestingly, a closed-form for the extinction probability is still available for this case.

Theorem 5.3. Suppose that $\rho_{b}<\rho_{c}<1$. Then, for any $i \geq 1$, the extinction probability $a_{i}$ of the IBCP starting from $i \geq 1$ is given by

$$
a_{i}=\frac{\int_{\zeta_{c}}^{\rho_{c}}\left(y^{i} A(y) / C(y)\right) \mathrm{d} y}{\int_{\zeta_{c}}^{\rho_{c}}(A(y) / C(y)) \mathrm{d} y} .
$$

Proof. Integrating (2.1) with respect to $t \in[0, \infty)$ yields

$$
\frac{C(s)}{2} G_{i}^{\prime \prime}(s)+B(s) G_{i}^{\prime}(s)=a_{i}-s^{i}, \quad i \geq 1,|s|<1 .
$$

Solving (5.2) on $\left[0, \rho_{c}\right)$ yields

$$
G_{i}^{\prime}(s) \exp \left\{\int_{0}^{s} \frac{2 B(x)}{C(x)} \mathrm{d} x\right\}-G_{i}^{\prime}(0)=\int_{0}^{s} \frac{2\left(a_{i}-y^{i}\right)}{C(y)} \exp \left\{\int_{0}^{y} \frac{2 B(x)}{C(x)} \mathrm{d} x\right\} \mathrm{d} y .
$$

Noting that $G_{i}^{\prime}\left(\rho_{c}\right)<+\infty$ and $\int_{0}^{\rho_{c}}(2 B(x) / C(x)) \mathrm{d} x=-\infty$ (since $B(x)<0$ for $\left.x \in\left(\rho_{b}, \rho_{c}\right)\right)$, we obtain

$$
-G_{i}^{\prime}(0)=\int_{0}^{\rho_{c}} \frac{2\left(a_{i}-y^{i}\right)}{C(y)} \exp \left\{\int_{0}^{y} \frac{2 B(x)}{C(x)} \mathrm{d} x\right\} \mathrm{d} y .
$$

Similarly, solving (5.2) on $\left(\zeta_{c}, 0\right]$ yields

$$
G_{i}^{\prime}(s) \exp \left\{\int_{0}^{s} \frac{2 B(x)}{C(x)} \mathrm{d} x\right\}-G_{i}^{\prime}(0)=\int_{0}^{s} \frac{2\left(a_{i}-y^{i}\right)}{C(y)} \exp \left\{\int_{0}^{y} \frac{2 B(x)}{C(x)} \mathrm{d} x\right\} \mathrm{d} y .
$$

Noting that $G_{i}^{\prime}\left(\zeta_{c}\right)<+\infty$ and $\int_{0}^{\zeta_{c}}(2 B(x) / C(x)) \mathrm{d} x=-\infty$ (since $B(x)>0$ for $\left.x \in\left(\zeta_{c}, 0\right)\right)$, we obtain

$$
-G_{i}^{\prime}(0)=\int_{0}^{\zeta_{c}} \frac{2\left(a_{i}-y^{i}\right)}{C(y)} \exp \left\{\int_{0}^{y} \frac{2 B(x)}{C(x)} \mathrm{d} x\right\} \mathrm{d} y .
$$

It follows from (5.3) and (5.4) that

$$
\int_{\zeta_{c}}^{\rho_{c}} \frac{2\left(a_{i}-y^{i}\right)}{C(y)} \exp \left\{\int_{0}^{y} \frac{2 B(x)}{C(x)} \mathrm{d} x\right\} \mathrm{d} y=0,
$$

and, thus, (5.1) follows.

Again, Kalinkin [10] obtained this theorem under the condition that $Q$ takes the special form given in Example 6.1 below.

Remark 5.1. By (5.1), we see that we have the following asymptotic behavior for the extinction probability $a_{i}$ :

$$
a_{i} \sim k i^{\alpha} \rho_{c}^{i} \quad \text { as } i \rightarrow+\infty
$$

where $k$ and $\alpha$ are two positive constants. Indeed, it is not difficult to show that the extinction probability could be rewritten as $a_{i}=k B(i+1, \alpha)$ for two positive constants $k$ and $\alpha$, where $B(a, b)$ is the beta function. Using the well-known result $\lim _{z \rightarrow+\infty} \Gamma(z+\alpha) z^{-\alpha} / \Gamma(z)=1$, once again, where $\Gamma(x)$ is the gamma function, we immediately obtain (5.5). A further detailed 
investigation shows that the constant $\alpha$ is just $2 B\left(\rho_{c}\right) / C^{\prime}\left(\rho_{c}\right)$, which is positive since both $B\left(\rho_{c}\right)$ and $C^{\prime}\left(\rho_{c}\right)$ are negative. Hence, we actually have

$$
a_{i} \sim k i^{2 B\left(\rho_{c}\right) / C^{\prime}\left(\rho_{c}\right)} \rho_{c}^{i} \quad \text { as } i \rightarrow+\infty .
$$

This interesting fact has already been observed by Kalinkin [10].

We now consider the subexplosive case. Surprisingly, this case is very subtle. To the authors' knowledge, for this subtle case, few results have been obtained until now. Even for the very special case given in Example 6.1 below, this is still an open problem. For example, this case was not considered in [10]. This seems to indicate that the subexplosive case is indeed challenging. Before we formally tackle this problem, we first show that a trivial lower bound as well as upper bound could be easily given.

Theorem 5.4. Suppose that the IBC q-matrix $Q$ is subexplosive, i.e. $\rho_{c}<\rho_{b} \leq 1$. Then $\rho_{c}^{i}<a_{i}<\rho_{b}^{i}(i \geq 1)$.

Proof. Letting $s=\rho_{c}$ and $\rho_{b}$ in (2.4) and using the dominated convergence theorem, together with Lemma 2.1, we immediately obtain the result. Note that, however, if $\rho_{c}<\rho_{b}=1$ then the fact that $a_{i}<\rho_{b}^{i}=1$ follows from the fact that the IBCP is not honest; see Theorem 3.1.

We could do much better than Theorem 5.4. To this end, we need to define the following simple transformation.

First, we rewrite the basic equation (4.3) as

$$
A_{0}(s) G_{i}^{\prime \prime}(s)+B_{0}(s) G_{i}^{\prime}(s)=U_{0}(s)
$$

where $A_{0}(s)=C(s) / 2, B_{0}(s)=B(s)$, and $U_{0}(s)=a_{i}-s^{i}$. Now if we define

$$
\begin{aligned}
& A_{1}(s)=A_{0}(s) B_{0}(s), \\
& B_{1}(s)=B_{0}(s)\left[B_{0}(s)+A_{0}^{\prime}(s)\right]-A_{0}(s) B_{0}^{\prime}(s), \\
& U_{1}(s)=B_{0}(s) U_{0}^{\prime}(s)-B_{0}^{\prime}(s) U_{0}(s),
\end{aligned}
$$

then we obtain

$$
A_{1}(s) G_{i}^{\prime \prime \prime}(s)+B_{1}(s) G_{i}^{\prime \prime}(s)=U_{1}(s) .
$$

Recursively, we could easily obtain, for any $n \geq 0$,

$$
A_{n}(s) G_{i}^{(n+2)}(s)+B_{n}(s) G_{i}^{(n+1)}(s)=U_{n}(s),
$$

where $\left\{A_{n}(s), B_{n}(s), U_{n}(s)\right\}$ are defined recursively as

$$
\begin{aligned}
& A_{n}(s)=A_{n-1}(s) B_{n-1}(s), \\
& B_{n}(s)=B_{n-1}(s)\left[B_{n-1}(s)+A_{n-1}^{\prime}(s)\right]-A_{n-1}(s) B_{n-1}^{\prime}(s), \\
& U_{n}(s)=B_{n-1}(s) U_{n-1}^{\prime}(s)-B_{n-1}^{\prime}(s) U_{n-1}(s) .
\end{aligned}
$$

Note that all the $A_{n}(s)$ and $B_{n}(s)(n \geq 0)$ are entirely expressible in terms of the given functions $B(s)$ and $C(s)$, and are also independent of $i \geq 1$. Similarly, all the $U_{n}(s)$ are totally expressible in terms of $B(s)$ and $C(s)$ together with the unknown constant $a_{i}$. In particular, all the $\left\{A_{n}(s), B_{n}(s), U_{n}(s)\right\}$ are power series of $s$ and, thus, analytic on the complex 
disk $\{z ;|z|<R\}$, where $R=R_{b} \wedge R_{c}$ and $R_{b}$ and $R_{c}$ are the convergence radii of the two power series $B(s)$ and $C(s)$, respectively. Of course, we have $R \geq 1$.

By (5.8) we immediately obtain

$$
A_{n}(s)=A_{0}(s) \prod_{k=0}^{n-1} B_{k}(s)
$$

Noting that $A_{0}(s)=C(s) / 2$ immediately yields

$$
A_{n}\left(\rho_{c}\right)=A_{n}\left(\zeta_{c}\right)=0 \text { for all } n \geq 0 .
$$

It then easily follows that

$$
\begin{gathered}
B_{n}\left(\rho_{c}\right)=\left(B_{0}\left(\rho_{c}\right)+n A_{0}^{\prime}\left(\rho_{c}\right)\right) \prod_{k=0}^{n-1} B_{k}\left(\rho_{c}\right) \quad \text { for all } n \geq 1, \\
A_{n}^{\prime}\left(\rho_{c}\right)=A_{0}^{\prime}\left(\rho_{c}\right) \prod_{k=0}^{n-1} B_{k}\left(\rho_{c}\right) \quad \text { for all } n \geq 1
\end{gathered}
$$

with

$$
B_{0}\left(\rho_{c}\right)=B\left(\rho_{c}\right)>0 \quad \text { and } \quad A_{0}^{\prime}\left(\rho_{c}\right)=\frac{C^{\prime}\left(\rho_{c}\right)}{2}<0
$$

Similarly,

$$
\begin{gathered}
B_{n}\left(\zeta_{c}\right)=\left(B_{0}\left(\zeta_{c}\right)+n A_{0}^{\prime}\left(\zeta_{c}\right)\right) \prod_{k=0}^{n-1} B_{k}\left(\zeta_{c}\right) \quad \text { for all } n \geq 1, \\
A_{n}^{\prime}\left(\zeta_{c}\right)=A_{0}^{\prime}\left(\zeta_{c}\right) \prod_{k=0}^{n-1} B_{k}\left(\zeta_{c}\right) \quad \text { for all } n \geq 1,
\end{gathered}
$$

with

$$
B_{0}\left(\zeta_{c}\right)=B\left(\zeta_{c}\right)>0 \quad \text { and } \quad A_{0}^{\prime}\left(\zeta_{c}\right)=\frac{C^{\prime}\left(\zeta_{c}\right)}{2}>0 .
$$

Regarding the signs of $B_{n}\left(\rho_{c}\right)$ and $B_{n}\left(\zeta_{c}\right)$ which are important in the later discussion, we have the following simple conclusions.

Lemma 5.1. Suppose that $Q$ is subexplosive. Then the following statements hold.

(i) $A_{n}\left(\rho_{c}\right)=A_{n}\left(\zeta_{c}\right)=0$ for all $n \geq 0$.

(ii) $B_{n}\left(\zeta_{c}\right)>0$ for all $n \geq 0$.

(iii) If $-2 B\left(\rho_{c}\right) / C^{\prime}\left(\rho_{c}\right)$ is a positive integer $m$, say, then $B_{n}\left(\rho_{c}\right)>0$ for all $0 \leq n \leq m-1$ and $B_{m}\left(\rho_{c}\right)=0$. If $-2 B\left(\rho_{c}\right) / C^{\prime}\left(\rho_{c}\right)$ is not a positive integer then $B_{n}\left(\rho_{c}\right)>0$ for all $0 \leq n<m$ and $B_{m}\left(\rho_{c}\right)<0$, where $m=\left[-2 B\left(\rho_{c}\right) / C^{\prime}\left(\rho_{c}\right)\right]$ is the integer part of $-2 B\left(\rho_{c}\right) / C^{\prime}\left(\rho_{c}\right)$.

Proof. The proof of part (i) was shown just above (5.11). Since $B_{0}\left(\zeta_{c}\right)>0$ and $A_{0}^{\prime}\left(\zeta_{c}\right)>0$, we can easily obtain (ii) by mathematical induction. Finally, (iii) follows directly from (5.11). 
We are now ready to provide more information about the extinction properties for the subexplosive case, i.e. $\rho_{c}<\rho_{b} \leq 1$. We first show that we could do much better than Theorem 5.1 by providing much narrower bounds.

Theorem 5.5. Suppose that $\rho_{c}<\rho_{b} \leq 1$.

(i) If $C^{\prime}\left(\rho_{c}\right)+2 B\left(\rho_{c}\right)=0$ then

$$
a_{i}=\rho_{c}^{i}+i \sigma \rho_{c}^{i-1}
$$

where the positive constant $\sigma$ is independent of $i$ and given by

$$
\sigma=-\frac{B\left(\rho_{c}\right)}{B^{\prime}\left(\rho_{c}\right)} .
$$

(ii) If $C^{\prime}\left(\rho_{c}\right)+2 B\left(\rho_{c}\right)>0$ then

$$
\rho_{c}^{i}+i \sigma \rho_{c}^{i-1}<a_{i}<\rho_{b}^{i},
$$

where $\sigma$ is the same as in (5.15).

(iii) If $C^{\prime}\left(\rho_{c}\right)+2 B\left(\rho_{c}\right)<0$ then

$$
\rho_{c}^{i}<a_{i}<\min \left\{\rho_{b}^{i}, \rho_{c}^{i}+i \sigma \rho_{c}^{i-1}\right\} .
$$

In particular, if $\rho_{b}=1$ then

$$
\rho_{c}^{i}<a_{i}<\rho_{c}^{i}+i \sigma \rho_{c}^{i-1} .
$$

Proof. Letting $n=1 \mathrm{in}(5.7)-(5.10)$ and using the facts that $B_{0}(s)=B(s), C_{0}(s)=C(s) / 2$, and $U_{0}(s)=a_{i}-s^{i}$, we see that

$$
\begin{aligned}
& \frac{C(s) B(s)}{2} G_{i}^{\prime \prime \prime}(s)+\frac{B(s)\left(2 B(s)+C^{\prime}(s)\right)-C(s) B^{\prime}(s)}{2} G_{i}^{\prime \prime}(s) \\
& =B^{\prime}(s)\left(s^{i}-a_{i}\right)-i s^{i-1} B(s)
\end{aligned}
$$

Letting $s=\rho_{c}$ in (5.16), noting that $C\left(\rho_{c}\right)=0$, and using the facts that $G_{i}^{\prime \prime \prime}\left(\rho_{c}\right)<\infty$ and $G_{i}^{\prime \prime}\left(\rho_{c}\right)<\infty$, we obtain

$$
\left[B\left(\rho_{c}\right)\left(2 B\left(\rho_{c}\right)+C^{\prime}\left(\rho_{c}\right)\right)\right] G_{i}^{\prime \prime}\left(\rho_{c}\right)=2 B^{\prime}\left(\rho_{c}\right)\left(\rho_{c}^{i}-a_{i}\right)-2 i \rho_{c}^{i-1} B\left(\rho_{c}\right) .
$$

Now, noting that $0<G_{i}^{\prime \prime}\left(\rho_{c}\right)<\infty$ and using some easy algebra, we obtain all the conclusions.

By Theorem 5.5 we see that if $C^{\prime}\left(\rho_{c}\right)+2 B\left(\rho_{c}\right)=0$ then the exact values of $a_{i}(i \geq 1)$ are given, while if $C^{\prime}\left(\rho_{c}\right)+2 B\left(\rho_{c}\right)<0$, only better bounds are provided. In fact, for the latter case, an explicit expression for $a_{i}(i \geq 1)$ is available.

Theorem 5.6. Suppose that the IBC q-matrix $Q$ is subexplosive and that $C^{\prime}\left(\rho_{c}\right)+2 B\left(\rho_{c}\right)<0$. Then we have

$$
\begin{aligned}
a_{i}= & \int_{\zeta_{c}}^{\rho_{c}} \frac{y^{i} B^{\prime}(y)-i y^{i-1} B(y)}{A_{1}(y)} \exp \left\{\int_{0}^{y} \frac{B_{1}(x)}{A_{1}(x)} \mathrm{d} x\right\} \mathrm{d} y \\
& \times\left(\int_{\zeta_{c}}^{\rho_{c}} \frac{B^{\prime}(y)}{A_{1}(y)} \exp \left\{\int_{0}^{y} \frac{B_{1}(x)}{A_{1}(x)} \mathrm{d} x\right\} \mathrm{d} y\right)^{-1} .
\end{aligned}
$$


Proof. By (5.17), we know that, for $|s|<\rho_{c}$,

$$
A_{1}(s) G_{i}^{\prime \prime \prime}(s)+B_{1}(s) G_{i}^{\prime \prime}(s)=U_{1}(s)
$$

where

$$
A_{1}(s)=\frac{C(s) B(s)}{2}, \quad B_{1}(s)=\frac{B(s)\left(2 B(s)+C^{\prime}(s)-C(s) B^{\prime}(s)\right)}{2},
$$

and

$$
U_{1}(s)=B^{\prime}(s)\left(s^{i}-a_{i}\right)-i s^{i-1} B(s) .
$$

Noting that $A_{1}(s)>0$ for all $s \in\left(\zeta_{c}, \rho_{c}\right)$ and $G^{\prime \prime}\left(\zeta_{c}\right) \exp \left\{\int_{0}^{\zeta_{c}}\left(B_{1}(y) / A_{1}(y)\right) \mathrm{d} y\right\}=0$, we can solve (5.18) to obtain

$$
G^{\prime \prime}(s) \exp \left\{\int_{0}^{s} \frac{B_{1}(y)}{A_{1}(y)} \mathrm{d} y\right\}=\int_{\zeta_{c}}^{s} \frac{U_{1}(y) \exp \left\{\int_{0}^{y}\left(B_{1}(x) / A_{1}(x)\right) \mathrm{d} x\right\}}{A_{1}(y)} \mathrm{d} y .
$$

As $B_{1}\left(\rho_{c}\right)=\frac{1}{2} B\left(\rho_{c}\right)\left(C^{\prime}\left(\rho_{c}\right)+2 B\left(\rho_{c}\right)\right)<0$, we have $G^{\prime \prime}\left(\rho_{c}\right) \exp \left\{\int_{0}^{\rho_{c}}\left(B_{1}(y) / A_{1}(y)\right) \mathrm{d} y\right\}=$ 0 . Therefore, letting $s \uparrow \rho_{c}$ in (5.19) yields

$$
\int_{\zeta_{c}}^{\rho_{c}} \frac{U_{1}(y) \exp \left\{\int_{0}^{y}\left(B_{1}(x) / A_{1}(x)\right) \mathrm{d} x\right\}}{A_{1}(y)} \mathrm{d} y=0
$$

i.e.

$$
\begin{aligned}
a_{i} \int_{\zeta_{c}}^{\rho_{c}} & \frac{B^{\prime}(y)}{A_{1}(y)} \exp \left\{\int_{0}^{y} \frac{B_{1}(x)}{A_{1}(x)} \mathrm{d} x\right\} \mathrm{d} y \\
\quad= & \int_{\zeta_{c}}^{\rho_{c}} \frac{y^{i} B^{\prime}(y)-i y^{i-1} B(y)}{A_{1}(y)} \exp \left\{\int_{0}^{y} \frac{B_{1}(x)}{A_{1}(x)} \mathrm{d} x\right\} \mathrm{d} y .
\end{aligned}
$$

Note that, since $B^{\prime}(y) \leq B^{\prime}(|y|) \leq B^{\prime}\left(\rho_{c}\right)<0$ for all $s \in\left(\zeta_{c}, \rho_{c}\right)$, it follows that

$$
\begin{aligned}
a_{i}= & \int_{\zeta_{c}}^{\rho_{c}} \frac{y^{i} B^{\prime}(y)-i y^{i-1} B(y)}{A_{1}(y)} \exp \left\{\int_{0}^{y} \frac{B_{1}(x)}{A_{1}(x)} \mathrm{d} x\right\} \mathrm{d} y \\
& \times\left(\int_{\zeta_{c}}^{\rho_{c}} \frac{B^{\prime}(y)}{A_{1}(y)} \exp \left\{\int_{0}^{y} \frac{B_{1}(x)}{A_{1}(x)} \mathrm{d} x\right\} \mathrm{d} y\right)^{-1} .
\end{aligned}
$$

The proof is complete.

By Theorems 5.5 and 5.6, we see that, for the closed form of the extinction probability, we only need to investigate the case in which $C^{\prime}\left(\rho_{c}\right)+2 B\left(\rho_{c}\right)>0$. In order to further examine this even subtler case, we need a little more algebra.

First, we need to know more about the structure of $U_{n}(s)$ defined in (5.10). Note that $U_{n}(s)$ depends upon $i \geq 1$. To emphasize this dependence, we will denote $U_{n}(s)$ as $U_{n i}(s)$.

Lemma 5.2. For any $n \geq 1$ and $i \geq 1$, we have

$$
U_{n i}(s)=\sum_{k=0}^{n} D_{n, k}(s) U_{0 i}^{(k)}(s),
$$

where $U_{0 i}^{(k)}(s)$ denotes the $k$ th derivative of $U_{0 i}(s)=a_{i}-s^{i}$, and $\left\{D_{n, k}(s) ; 0 \leq k \leq n\right\}$ are totally expressible by the two known functions $B(s)$ and $C(s)$. In particular, $D_{n, k}(s)$ does not 
depend on $i \geq 1$. Moreover, $\left\{D_{n, k}(s)\right\}$ can be given recursively as

$$
\begin{gathered}
D_{1,0}(s)=-B^{\prime}(s), \quad D_{1,1}(s)=B(s), \\
D_{n, k}(s)=D_{n-1, k-1}(s) B_{n-1}(s)-D_{n-1, k}(s) B_{n-1}^{\prime}(s) \\
+D_{n-1, k}^{\prime}(s) B_{n-1}(s), \quad k \leq n-1,
\end{gathered}
$$

and

$$
D_{n, n}(s)=\prod_{m=0}^{n-1} B_{m}(s)
$$

Proof. Applying the induction principle to (5.10) immediately yields all the conclusions.

Remark 5.2. By the definition of $U_{0 i}(s)$ we see that $U_{n i}(s)=\sum_{k=0}^{n \wedge i} D_{n, k}(s) U_{0 i}^{(k)}(s)$. However, for notational convenience, we will continue to use the form given in (5.20).

We now further consider the case in which $C^{\prime}\left(\rho_{c}\right)+2 B\left(\rho_{c}\right)>0$. Considering $B\left(\rho_{c}\right)>0$ and $C^{\prime}\left(\rho_{c}\right)<0$, there exists a positive integer $m$ such that

$$
m C^{\prime}\left(\rho_{c}\right)+2 B\left(\rho_{c}\right) \leq 0 \text { and }(m-1) C^{\prime}\left(\rho_{c}\right)+2 B\left(\rho_{c}\right)>0 .
$$

In Theorems 5.5 and 5.6 we addressed the $m=1$ case. We now consider the general case for $m \geq 2$.

Theorem 5.7. Suppose that $Q$ is a subexplosive IBC $q$-matrix with $C^{\prime}\left(\rho_{c}\right)+2 B\left(\rho_{c}\right)>0$. Let $m=\min \left\{k \in \mathbb{Z}_{+} ; k C^{\prime}\left(\rho_{c}\right)+2 B\left(\rho_{c}\right) \leq 0\right\}$ and, thus, $m \geq 2$.

(i) If $m C^{\prime}\left(\rho_{c}\right)+2 B\left(\rho_{c}\right)=0$ then $U_{k}\left(\rho_{c}\right)>0$ for all $0 \leq k \leq m-1$ and $U_{m}\left(\rho_{c}\right)=0$. Hence,

$$
a_{i}=\rho_{c}^{i}+\sum_{k=1}^{m \wedge i} \frac{D_{m, k}\left(\rho_{c}\right)}{D_{m, 0}\left(\rho_{c}\right)} \frac{i !}{(i-k) !} \rho_{c}^{i-k} .
$$

In particular, $a_{1}=\rho_{c}+D_{m, 1}\left(\rho_{c}\right) / D_{m, 0}\left(\rho_{c}\right)$.

(ii) If $m C^{\prime}\left(\rho_{c}\right)+2 B\left(\rho_{c}\right)<0$ then $U_{k}\left(\rho_{c}\right)>0$ for all $0 \leq k \leq m-1$ and $U_{m}\left(\rho_{c}\right)<0$. Hence,

$$
\rho_{c}^{i}+\sum_{k=1}^{(m-1) \wedge i} \frac{D_{m-1, k}\left(\rho_{c}\right)}{D_{m-1,0}\left(\rho_{c}\right)} \frac{i !}{(i-k) !} \rho_{c}^{i-k}<a_{i}<\rho_{c}^{i}+\sum_{k=1}^{m \wedge i} \frac{D_{m, k}\left(\rho_{c}\right)}{D_{m, 0}\left(\rho_{c}\right)} \frac{i !}{(i-k) !} \rho_{c}^{i-k} .
$$

In particular, $\rho_{c}+D_{m-1,1}\left(\rho_{c}\right) / D_{m-1,0}\left(\rho_{c}\right)<a_{1}<\rho_{c}+D_{m, 1}\left(\rho_{c}\right) / D_{m, 0}\left(\rho_{c}\right)$.

Proof. It follows from (5.11) that

$$
B_{k}\left(\rho_{c}\right)=\frac{1}{2}\left(2 B\left(\rho_{c}\right)+k C^{\prime}\left(\rho_{c}\right)\right) \prod_{j=0}^{k-1} B_{j}\left(\rho_{c}\right) \quad \text { for all } k \geq 1 .
$$

From (5.25) and $B_{0}\left(\rho_{c}\right)=B\left(\rho_{c}\right)>0$, we can easily see that

$$
m=\min \left\{k \geq 1 ; k C^{\prime}\left(\rho_{c}\right)+2 B\left(\rho_{c}\right) \leq 0\right\}=\min \left\{k \geq 1 ; B_{k}\left(\rho_{c}\right) \leq 0\right\} .
$$


Recall that we have

$$
A_{m}(s) G_{i}^{(m+2)}(s)+B_{m}(s) G_{i}^{(m+1)}(s)=U_{m}(s) .
$$

Letting $s=\rho_{c}$ in the above equation immediately yields $U_{m}\left(\rho_{c}\right)=0$. Then (5.23) immediately follows from (5.25) and Lemma 5.2. Finally, if $m C^{\prime}\left(\rho_{c}\right)+2 B\left(\rho_{c}\right)<0$, we may similarly prove that $U_{k}\left(\rho_{c}\right)>0$ for all $0 \leq k \leq m-1$ and $U_{m}\left(\rho_{c}\right)<0$, and, hence, the inequalities in (5.24) follow.

Remark 5.3. In obtaining (5.23) we assumed that $D_{m, 0}\left(\rho_{c}\right) \neq 0$. Indeed, if $D_{m, 0}\left(\rho_{c}\right)=0$ then by letting $s=\rho_{c}$ in (5.20) for $i=1$ (see also Remark 5.2) we would obtain $D_{m, 1}\left(\rho_{c}\right)=0$. Now using (5.20) (again, referring to Remark 5.2) for $i=2,3, \ldots$, etc., we obtain, for all $i \in \mathbb{Z}_{+}, D_{m, i}\left(\rho_{c}\right)=0$. This implies that both $A_{m}(s)$ and $B_{m}(s)$ are divisible by $s-\rho_{c}$ (see (5.21) and (5.22)), and, thus, the equation $A_{m}(s) G_{i}^{(m+2)}(s)+B_{m}(s) G_{i}^{(m+1)}(s)=U_{m}(s)$ could be reduced by dividing $s-\rho_{c}$. Similarly, in obtaining (5.24) we further assumed that $D_{m-1,0}\left(\rho_{c}\right)>0$ and $D_{m, 0}\left(\rho_{c}\right)>0$. If they are negative, inequality (5.24) may need to be reversed.

Remark 5.4. By (5.15) and (5.23), it is easily seen that if $m C^{\prime}\left(\rho_{c}\right)+2 B\left(\rho_{c}\right)=0$ then we still have the following asymptotic behavior for the extinction probability $a_{i}$ :

$$
a_{i} \sim k i^{\alpha} \rho_{c}^{i} \quad \text { as } i \rightarrow+\infty .
$$

Here $k$ is a constant and $\alpha=m=-2 B\left(\rho_{c}\right) / C^{\prime}\left(\rho_{c}\right)$. Comparing this with (5.6) in Remark 5.1, shows that $2 B\left(\rho_{c}\right) / C^{\prime}\left(\rho_{c}\right)$ is a key quantity in studying the extinction behavior of the IBCP process. Furthermore, inequality (5.24) has already provided a strong hint that (5.26) may still hold true with $\alpha=-2 B\left(\rho_{c}\right) / C^{\prime}\left(\rho_{c}\right)$ when $m C^{\prime}\left(\rho_{c}\right)+2 B\left(\rho_{c}\right)<0$. We will investigate such interesting behavior in a subsequent paper.

Although Theorem 5.7 (ii) yields much better bounds for the extinction probabilities, closed forms are not given. In order to obtain such closed forms, we will suppose that Assumption A below holds. Later we will see that in some sense the purpose of this assumption is just to simplify the notation. Recall that we only need to consider the case in which $C^{\prime}\left(\rho_{c}\right)+2 B\left(\rho_{c}\right)>$ 0 , since otherwise the answer has already been given in Theorem 5.6. We will also assume that $-2 B\left(\rho_{c}\right) / C^{\prime}\left(\rho_{c}\right)$ is not a positive integer, since otherwise the problem is also solved; see Theorem 5.7. Now considering $C^{\prime}\left(\rho_{c}\right)<0$ and $B\left(\rho_{c}\right)>0$, we could find the smallest positive integer $k>1$ such that $k C^{\prime}\left(\rho_{c}\right)+2 B\left(\rho_{c}\right)<0$.

Assumption A. For a subexplosive IBC $q$-matrix $Q$ satisfying $C^{\prime}\left(\rho_{c}\right)+2 B\left(\rho_{c}\right)>0$, we assume that $A_{m}(s)>0$ for all $s \in\left(\zeta_{c}, \rho_{c}\right)$, where $m=\min \left\{k \geq 1 ; k C^{\prime}\left(\rho_{c}\right)+2 B\left(\rho_{c}\right)<0\right\}$ and $A_{m}(s)$ is given in (5.10).

We now show that, under Assumption A, closed-form extinction probabilities are available.

Theorem 5.8. Suppose that $Q$ is a subexplosive IBC $q$-matrix satisfying $C^{\prime}\left(\rho_{c}\right)+2 B\left(\rho_{c}\right)>0$ and that $-2 B\left(\rho_{c}\right) / C^{\prime}\left(\rho_{c}\right)$ is not an integer. Let $m=\min \left\{k \geq 1 ; k C^{\prime}\left(\rho_{c}\right)+2 B\left(\rho_{c}\right)<0\right\}$. Furthermore, assume that $A_{m}(s)$ satisfies Assumption A. Then

$$
a_{i}=\sum_{k=0}^{m \wedge i} \frac{i !}{(i-k) !} \int_{\zeta_{c}}^{\rho_{c}} \frac{y^{i-k} D_{m, k}(y)}{A_{m}(y)} \mathrm{e}^{H_{m}(y)} \mathrm{d} y / \int_{\zeta_{c}}^{\rho_{c}} \frac{D_{m, 0}(y)}{A_{m}(y)} \mathrm{e}^{H_{m}(y)} \mathrm{d} y,
$$

where $H_{m}(y)=\int_{0}^{y}\left(B_{m}(x) / A_{m}(x)\right) \mathrm{d} x$ and $D_{m, k}(s)$ etc. are given in Lemma 5.2. 
Proof. Consider (5.7) with $n=m$, i.e.

$$
A_{m}(s) G_{i}^{(m+2)}(s)+B_{m}(s) G_{i}^{(m+1)}(s)=U_{m i}(s) .
$$

Solving the above equation under Assumption A yields

$$
G_{i}^{(m+1)}(s) \mathrm{e}^{H_{m}(s)}-G_{i}^{(m+1)}(0)=\int_{0}^{s} \frac{U_{m i}(y)}{A_{m}(y)} \mathrm{e}^{H_{m}(y)} \mathrm{d} y .
$$

Since we only need to consider the case stated in Theorem 5.7(ii) and, thus, by the definition of $m$, we know that $B_{n}\left(\rho_{c}\right)>0$ for all $0 \leq n \leq m-1$ and $B_{m}\left(\rho_{c}\right)<0$. Hence, $B_{m}(s)<0$ in a sufficiently small interval $\left(\rho_{c}-\varepsilon, \rho_{c}\right)$ (where $\varepsilon>0$ ). This, together with Assumption A, implies that $\mathrm{e}^{H_{m}(s)} \rightarrow 0$ as $s \rightarrow \rho_{c}^{-}$since $A_{m}\left(\rho_{c}\right)=0$. Now using the fact that $0<G_{i}^{(m+1)}\left(\rho_{c}\right)<+\infty$ and letting $s \rightarrow \rho_{c}^{-}$in (5.28), we immediately obtain

$$
-G_{i}^{(m+1)}(0)=\int_{0}^{\rho_{c}} \frac{U_{m i}(y)}{A_{m}(y)} \mathrm{e}^{H_{m}(y)} \mathrm{d} y .
$$

On the other hand, by looking at (5.12)-(5.14), we know that $\mathrm{e}^{H_{m}(s)} \rightarrow 0$ as $s \rightarrow \zeta_{c}^{+}$; thus, letting $s \rightarrow \zeta_{c}^{+}$in (5.28) and, again, using the fact that $\left|G_{i}^{(m+1)}\left(\xi_{c}\right)\right|<+\infty$ yield

$$
-G_{i}^{(m+1)}(0)=\int_{0}^{\zeta_{c}} \frac{U_{m i}(y)}{A_{m}(y)} \mathrm{e}^{H_{m}(y)} \mathrm{d} y .
$$

By combining the above proven two equalities, we obtain

$$
\int_{\zeta_{c}}^{\rho_{c}} \frac{U_{m i}(y)}{A_{m}(y)} \mathrm{e}^{H_{m}(y)} \mathrm{d} y=0 .
$$

Now, substituting (5.20) into the above equality immediately yields (5.27).

We now consider the situation in which Assumption A fails. Recall that $A_{m}(s)$ is an analytic function of $s$ and, thus, has only finitely many zeros in $\left(\zeta_{c}, \rho_{c}\right)$. Without loss of generality, we assume that 0 is not a zero of $A_{m}(s)$, since otherwise we could choose another point which is not a zero of $A_{m}(s)$ as the starting point of our following discussion. Let $\mu_{0}$ be the smallest negative zero of $A_{m}(s)$ on $\left(\zeta_{c}, \rho_{c}\right)$ (if $A_{m}(s)$ has no negative zero on $\left(\zeta_{c}, \rho_{c}\right)$, we could simply let $\left.\mu_{0}=0\right)$. Now, for each negative zero $\mu_{k}$, say, of $A_{m}(s)$, we could find a sufficiently small radius $c_{k}$ such that $A_{m}(s)$ has no zero on the disk $\left\{z ;\left|z-\mu_{k}\right| \leq c_{k}\right\}$ except $\mu_{k}$ itself. Now, for any $y$ such that $\zeta_{c}<y<\mu_{0}$ and any analytic function $f(s)$, we could define the complex integral $\oint_{C_{y}}\left(f(s) / A_{m}(s)\right) \mathrm{d} s$, where the closed curve $C_{y}$ is formed as follows: it starts at $y$, moving to the right along the real axis until reaching 0 and then moving back to the left, again, along the real axis until reaching and then ending at $y$, and whenever it hits a circle $\left\{z ;\left|z-\mu_{k}\right|=c_{k}\right\}$ as defined above, it moves clockwise along the upper half of the circle first and then (when returning leftwise) moves clockwise along the other half of the circle. Now this integral is a real value. Indeed, by the residue theorem in complex analysis, this integral just equals the sum of residues of the function $f(s) / A_{m}(s)$ at zeros of $A_{m}(s)$. By the symmetric property, we know that the integral $\int_{\tilde{C}_{y}}\left(f(s) / A_{m}(s)\right) \mathrm{d} s$, where $\tilde{C}_{y}$ is just the upper part of $C_{y}$, starting from 0 and ending at $y$, is just half of the above value and, thus, is again a real value. As a convention, in the following we will simply use $(\sim) \oint_{0}^{y}\left(f(s) / A_{m}(s)\right) \mathrm{d} s$ to denote this integral. Of course, this integral does not depend on the above disk $\left\{z ;\left|z-\mu_{k}\right| \leq c_{k}\right\}$. If necessary, we could use 
any other curve $D_{y}$, say, so long as the closed curve, formed by $C_{y}$ and $D_{y}$, does not contain any zeros of $A_{m}(s)$. Similarly, let $\lambda_{0}$ be the largest positive zero of $A_{m}(s)$ on $\left(\zeta_{c}, \rho_{c}\right)$. Then, for any $\lambda_{0}<y<\rho_{c}$ and any analytic function $f(s)$, the integral $(\sim) \oint_{0}^{y}\left(f(s) / A_{m}(s)\right) \mathrm{d} s$ is also well defined and is real valued since this integral does not pass through any zero of $A_{m}(s)$. Now, by such convention and understanding, we obtain the following conclusion, which takes a similar form to Theorem 5.8.

Theorem 5.9. Suppose that $Q$ is a subexplosive IBC $q$-matrix satisfying $C^{\prime}\left(\rho_{c}\right)+2 B\left(\rho_{c}\right)>0$ and that $-2 B\left(\rho_{c}\right) / C^{\prime}\left(\rho_{c}\right)$ is not an integer. Let $m=\min \left\{k \geq 1 ; k C^{\prime}\left(\rho_{c}\right)+2 B\left(\rho_{c}\right)<0\right\}$. Furthermore, assume that $A_{m}(s)$ does not satisfy Assumption A. Then

$$
a_{i}=\sum_{k=0}^{m \wedge i} \frac{i !}{(i-k) !}(\sim) \oint_{\zeta_{c}}^{\rho_{c}} \frac{y^{i-k} D_{m, k}(y)}{A_{m}(y)} \mathrm{e}^{H_{m}(y)} \mathrm{d} y /(\sim) \oint_{\zeta_{c}}^{\rho_{c}} \frac{D_{m, 0}(y)}{A_{m}(y)} \mathrm{e}^{H_{m}(y)} \mathrm{d} y,
$$

where $H_{m}(y)=(\sim) \oint_{0}^{y}\left(B_{m}(x) / A_{m}(x)\right) \mathrm{d} x$.

Proof. Again, consider (5.7) with $n=m$, i.e.

$$
A_{m}(s) G_{i}^{(m+2)}(s)+B_{m}(s) G_{i}^{(m+1)}(s)=U_{m i}(s) .
$$

However, we now view it as a complex-valued differential equation with complex-valued coefficient functions $A_{m}(s)$ and $B_{m}(s)$ etc. It is easily seen that this equation does make sense using the above interpretation. Now, solving the above equation, we still obtain

$$
G_{i}^{(m+1)}(s) \mathrm{e}^{H_{m}(s)}-G_{i}^{(m+1)}(0)=(\sim) \oint_{0}^{s} \frac{U_{m i}(y)}{A_{m}(y)} \mathrm{e}^{H_{m}(y)} \mathrm{d} y,
$$

in the sense defined above. By reasoning similar to that used to prove Theorem 5.8, if we let $s \rightarrow \rho_{c}^{-}$in (5.30), we immediately obtain

$$
-G_{i}^{(m+1)}(0)=(\sim) \oint_{0}^{\rho_{c}} \frac{U_{m i}(y)}{A_{m}(y)} \mathrm{e}^{H_{m}(y)} \mathrm{d} y .
$$

Similarly, we could obtain

$$
-G_{i}^{(m+1)}(0)=(\sim) \oint_{0}^{\zeta_{c}} \frac{U_{m i}(y)}{A_{m}(y)} \mathrm{e}^{H_{m}(y)} \mathrm{d} y .
$$

By combining the above two equalities, we may, again, obtain

$$
(\sim) \oint_{\zeta_{c}}^{\rho_{c}} \frac{U_{m i}(y)}{A_{m}(y)} \mathrm{e}^{H_{m}(y)} \mathrm{d} y=0 .
$$

Now, substituting (5.20) into the above equality immediately yields (5.29).

Therefore, in some sense, our Assumption A simply guarantees that (5.29) could take a simple form. In other words, Assumption A is not a serious limitation, it is simply notationally convenient.

Let's consider more details about the case of $m=2$ under the condition, of course, that $C^{\prime}\left(\rho_{c}\right)+2 B\left(\rho_{c}\right)>0$. To this end, we need more information about the properties of the function $B_{1}(s)$ which are shown below. 
Lemma 5.3. (i) For all $s \in\left(-1, \rho_{b}\right)$, and, in particular, for all $s \in\left(\zeta_{c}, \rho_{c}\right)$, we have $B(s)>0$.

(ii) For all $s \in\left(\zeta_{c}, \rho_{c}\right)$, we have $C(s)>0$.

(iii) For all $s \in\left(-\rho_{c}, \rho_{c}\right)$, and, in particular, for all $s \in\left[\zeta_{c}, \rho_{c}\right]$, we have $B^{\prime}(s)<0$.

(iv) The function $C^{\prime}(z)$, viewed as a complex function well defined at least on the unit disc, has exactly one zero in the disc $\left\{z ;|z| \leq \rho_{c}\right\}$ and this unique zero, denoted by $\eta_{1}$, is real and nonnegative. In particular, we have, for all $s \in\left[\zeta_{c}, \eta_{1}\right), C^{\prime}(s)>0$, while, for all $s \in\left(\eta_{1}, \rho_{c}\right]$, $C^{\prime}(s)<0$.

Proof. Parts (i) and (ii) are straightforward and have been proved in Lemma 2.1. Part (iii) is also straightforward. Indeed, using the fact that $B^{\prime}\left(\rho_{c}\right)<0$ together with $B^{\prime}(s)=\sum_{j=1}^{\infty} j b_{j} \times$ $s^{j-1}$, we obtain $\sum_{j=2}^{\infty} j b_{j} \rho_{c}^{j-1}<-b_{1}$. It follows that, for all $z \in\left\{z ;|z| \leq \rho_{c}\right\}$, we have $\left|\sum_{j=2}^{\infty} j b_{j} z^{j-1}\right| \leq \sum_{j=2}^{\infty} j b_{j} \rho_{c}^{j-1}<-b_{1}$, and, thus, $B^{\prime}(z)$ has no zero in the complex disc $\left\{z ;|z| \leq \rho_{c}\right\}$ and, in particular, no zero in the interval $\left[-\rho_{c}, \rho_{c}\right]$. But $B^{\prime}(0)=b_{1}<0$ and, thus, for all $s \in\left[-\rho_{c}, \rho_{c}\right]$, particularly for $s \in\left[\zeta_{c}, \rho_{c}\right], B^{\prime}(s)$ remains of negative sign.

To show (iv), we apply Rouche's theorem. To this end, define $f(z)=2 c_{2} z$ and $g(z)=$ $\sum_{j=1}^{\infty} j c_{j} z^{j-1}-2 c_{2} z$, and, thus, $f(z)+g(z)=C^{\prime}(z)$. Now it is easily seen that both $f(z)$ and $g(z)$ are analytic on the open disc $\left\{z ;|z|<\rho_{c}\right\}$ and continuous on the circle $\left\{z ;|z|=\rho_{c}\right\}$ (in fact, they are even analytic on the unit disc $\{z ;|z|<1\}$ ). Moreover, we could easily prove that, on the circle $\left\{z ;|z|=\rho_{c}\right\}$,

$$
|f(z)|>|g(z)|>0 .
$$

Indeed, firstly, the fact that $|g(z)|=\left|\sum_{j \neq 2} j c_{j} z^{j-1}\right|>0$ is clearly true on $\left\{z ;|z|=\rho_{c}\right\}$ since all the $c_{j}(j \neq 2)$ are nonnegative and at least one of them is strictly positive. Secondly, since $C^{\prime}\left(\rho_{c}\right)<0$, we know that $|g(z)| \leq \sum_{j \neq 2} j c_{j}|z|^{j-1} \leq \sum_{j \neq 2} j c_{j} \rho_{c}^{j-1}<-2 c_{2} \rho_{c}=|f(z)|$ and, thus, (5.31) is proven. Now, by Rouche's theorem we know that $C^{\prime}(z)$ and $f(z)$ have the same number of zeros on $\left\{z ;|z| \leq \rho_{c}\right\}$. But $f(z)=2 c_{2} z$ has clearly one zero on $\left\{z ;|z| \leq \rho_{c}\right\}$ and, thus, so does $C^{\prime}(z)$. Finally, considering $C^{\prime}(0)=c_{1} \geq 0$ and $C^{\prime}\left(\rho_{c}\right)<0$, this unique zero must be nonnegative. Now, if $c_{1}>0$ then all the conclusions in (iv) have been proven. If $C^{\prime}(0)=c_{1}=0$, we need to show that, for all $s \in\left[\zeta_{c}, 0\right)$, we have $C^{\prime}(s)>0$. But this is also easy. Indeed, it follows from the fact that $\lim _{s \uparrow 0^{-}} C^{\prime}(s) / s=2 c_{2}<0$.

The following simple lemma now easily follows.

Lemma 5.4. For $B_{1}(s) \equiv B(s)\left(B(s)+1 / 2 C^{\prime}(s)\right)-1 / 2 C(s) B^{\prime}(s)$, we have the following conclusions.

(i) For all $s \in\left[\zeta_{c}, 0\right]$, we have $B_{1}(s)>0$.

(ii) Either $B_{1}(s)>0$ for all $s \in\left[0, \rho_{c}\right]$, or $B_{1}(s)$ has exactly two positive zeros $0<\lambda_{1}<$ $\lambda_{2} \leq \rho_{c}$ such that $B_{1}(s)>0$ for all $s \in\left[0, \lambda_{1}\right) \cup\left(\lambda_{2}, \rho_{c}\right]$ and $B_{1}(s)<0$ for all $s \in\left(\lambda_{1}, \lambda_{2}\right)$. Moreover, the latter case occurs if and only if $\min \left\{B_{1}(s) ; s \in\left[0, \rho_{c}\right]\right\}<0$.

Proof. Considering $B_{1}(s)=B(s)\left(B(s)+1 / 2 C^{\prime}(s)\right)-1 / 2 C(s) B^{\prime}(s)$, (i) immediately follows from Lemma 5.3. For (ii), by Lemma 5.3 we first know that, for all $s \in\left[0, \rho_{c}\right]$, we have $-1 / 2 C(s) B^{\prime}(s)>0$ together with $B(s)>0$. Hence, for any $s \in\left(0, \rho_{c}\right)$, we have $B_{1}(s)<0$ if and only if

$$
B(s)+\frac{1}{2} C^{\prime}(s)<\frac{C(s) B^{\prime}(s)}{2 B(s)},
$$


where the right-hand side of (5.32) is negative. However, $B(s)+1 / 2 C^{\prime}(s)$ is a convex function on $[0,1]$ and, thus, can have at most two zeros. Since $B(0)+1 / 2 C^{\prime}(0)=b_{0}+1 / 2 c_{1}>0$, if $B(s)+1 / 2 C^{\prime}(s)$ has no zero or has only one zero on $(0,1]$, then $(5.32)$ cannot hold and, thus, $B_{1}(s)>0$ for all $s \in\left[0, \rho_{c}\right]$.

On the other hand, if $B(s)+1 / 2 C^{\prime}(s)$ has two zeros on $(0,1]$ then, in order for $(5.32)$ to hold, these two zeros must be in $\left(0, \rho_{c}\right]$, due to the facts that $B(0)+1 / 2 C^{\prime}(0)>0$ and $C\left(\rho_{c}\right)=0$. Now the first part of (ii) follows. Finally, if there does exist two zeros $\lambda_{1}<\lambda_{2}$ such that $B_{1}(s)<0$ for all $s \in\left(\lambda_{1}, \lambda_{2}\right)$, then clearly $\min \left\{B_{1}(s) ; s \in\left[0, \rho_{c}\right]\right\}<0$ and vice versa.

Remark 5.5. Lemma 5.4 provides a method to check whether $B_{1}(s)$ has two zeros or not in $\left[0, \rho_{c}\right]$. But such a calculation may be complex since finding the root of $B_{1}^{\prime}(s)$ may not be easy. The following relatively easy algebra could suffice. First find the zero of $B^{\prime}(s)+1 / 2 C^{\prime \prime}(s)$ on $\left[0, \rho_{c}\right]$, which must be unique, denoted by $\tau$, say. Then calculate $B_{1}(\tau)$. If $B_{1}(\tau)<0$ then we must have $\min \left\{B_{1}(s) ; s \in\left[0, \rho_{c}\right]\right\}<0$. However, the converse may not be true.

We now have the following conclusion whose proof is essentially given above.

Theorem 5.10. Suppose that $Q$ is a subexplosive IBC $q$-matrix satisfying of $C^{\prime}\left(\rho_{c}\right)+2 B\left(\rho_{c}\right)>$ 0 and $2 C^{\prime}\left(\rho_{c}\right)+2 B\left(\rho_{c}\right)<0$. Then we have the following conclusions.

(i) The equation $B(s)+1 / 2 C^{\prime}(s)=0$ either has no root in the interval $\left(0, \rho_{c}\right)$ or has exactly two roots in the interval $\left(0, \rho_{c}\right)$.

(ii) If $B(s)+1 / 2 C^{\prime}(s)=0$ has no root in $\left(0, \rho_{c}\right)$ or if $B(s)+1 / 2 C^{\prime}(s)=0$ does have two roots in $\left(0, \rho_{c}\right)$ but $\min \left\{B_{1}(s) ; s \in\left[0, \rho_{c}\right]\right\}>0$, then

$$
a_{1}=\int_{\zeta_{c}}^{\rho_{c}} \frac{y D_{2,0}(y)+D_{2,1}(y)}{A_{2}(y)} \mathrm{e}^{H_{2}(y)} \mathrm{d} y / \int_{\zeta_{c}}^{\rho_{c}} \frac{D_{2,0}(y)}{A_{2}(y)} \mathrm{e}^{H_{2}(y)} \mathrm{d} y,
$$

and, for $i \geq 2$,

$$
\begin{aligned}
a_{i}= & \int_{\zeta_{c}}^{\rho_{c}} \frac{y^{i} D_{2,0}(y)+i y^{i-1} D_{2,1}(y)+i(i-1) y^{i-2} D_{2,2}(y)}{A_{2}(y)} \mathrm{e}^{H_{2}(y)} \mathrm{d} y \\
& \times\left(\int_{\zeta_{c}}^{\rho_{c}} \frac{D_{2,0}(y)}{A_{2}(y)} \mathrm{e}^{H_{2}(y)} \mathrm{d} y\right)^{-1} .
\end{aligned}
$$

(iii) If $B(s)+1 / 2 C^{\prime}(s)=0$ has two roots in $\left(0, \rho_{c}\right)$ and $\min \left\{B_{1}(s) ; s \in\left[0, \rho_{c}\right]\right\}<0$, then

$$
a_{1}=(\sim) \oint_{\zeta_{c}}^{\lambda_{2}} \frac{y D_{2,0}(y)+D_{2,1}(y)}{A_{2}(y)} \mathrm{e}^{H_{2}(y)} \mathrm{d} y /(\sim) \oint_{\zeta_{c}}^{\lambda_{2}} \frac{D_{2,0}(y)}{A_{2}(y)} \mathrm{e}^{H_{2}(y)} \mathrm{d} y,
$$

and, for $i \geq 2$,

$$
\begin{aligned}
a_{i}= & (\sim) \oint_{\zeta_{c}}^{\lambda_{2}} \frac{y^{i} D_{2,0}(y)+i y^{i-1} D_{2,1}(y)+i(i-1) y^{i-2} D_{2,2}(y)}{A_{2}(y)} \mathrm{e}^{H_{2}(y)} \mathrm{d} y \\
& \times\left((\sim) \oint_{\zeta_{c}}^{\lambda_{2}} \frac{D_{2,0}(y)}{A_{2}(y)} \mathrm{e}^{H_{2}(y)} \mathrm{d} y\right)^{-1} .
\end{aligned}
$$

We end this section with the following important remark. 
Remark 5.6. Careful readers may have already noticed that nearly all the conclusions obtained in this section are based on the condition $\rho_{c}<+\infty$ only, rather than on the assumption that $0<C^{\prime}(1)<+\infty$ as stated at the beginning of this section. Indeed, it is easily seen that Theorems 5.1-5.3 and Theorems 5.5-5.10 still hold and all the proofs remain valid if $C^{\prime}(1)=+\infty$. Even Theorem 5.4 still holds (but the proofs need to be revised). In fact, the main reason in making the assumption that $0<C^{\prime}(1)<+\infty$ is for convenience only. Indeed, if $C^{\prime}(1)=+\infty$ and $B^{\prime}(1)=+\infty$, then the uniqueness question has not been fully resolved, see Section 3, and, thus, we are unable to firmly claim that we are dealing with the irregular case in this section.

\section{Examples}

Example 6.1. We first give a simple example where the birth structure for both the branching and collision components takes a single birth form. That is, we assume that

$$
b_{0}=a>0, \quad b_{1}=-(a+b), \quad b_{2}=b>0, \quad b_{j} \equiv 0 \quad \text { for all } j \geq 3,
$$

and that

$$
\begin{aligned}
& c_{0}=d>0, c_{1}=r \geq 0, \quad c_{2}=-(d+r+c), \\
& c_{3}=c>0, \quad c_{j} \equiv 0 \quad \text { for all } j \geq 4 .
\end{aligned}
$$

Note that this special example is just the one considered in Kalinkin (2003). Clearly, for this example, we have

$$
B(s)=a-(a+b) s+b s^{2}=a(1-s)\left(1-\frac{b s}{a}\right)
$$

and

$$
C(s)=d+r s-(d+r+c) s^{2}+c s^{3}=c(s-1)(s-q)(s-\zeta),
$$

where $q=\left(d+r+\sqrt{(d+r)^{2}+4 d c}\right) / 2 c$ and $\zeta=\left(d+r-\sqrt{(d+r)^{2}+4 d c}\right) / 2 c<0$. It is easily seen that $C^{\prime}(1)=c-(2 d+r)$ and $B^{\prime}(1)=b-a$.

By Theorem 4.1 and Corollary 4.1, we know that if $c<2 d+r$ or if $c=2 d+r$ and $b \leq a$, then extinction will definitely occur, that is, for all $i \geq 1, a_{i}=1$. We now turn our attention to the interesting case of $C^{\prime}(1)=0$ together with $B^{\prime}(1)>0$, or, equivalently, $c=2 d+r$ and $b>a$. For this case, easy algebra show that the discriminant quantity $J_{0}$ given in Remark 4.1 takes the form

$$
\begin{aligned}
J_{0} & =\int_{0}^{1} \frac{A(y)}{C(y)} \mathrm{d} y \\
& =\frac{1}{d} \int_{0}^{1}\left[1+\left(2+\frac{r}{d}\right) y\right]^{2(b d+a r+2 a d) /(3 d+r)(2 d+r)-1}(1-y)^{2(b-a) /(3 d+r)-2} \mathrm{~d} y .
\end{aligned}
$$

In particular, if $r=0$ then $J_{0}$ takes the very simple form

$$
J_{0}=\int_{0}^{1} \frac{A(y)}{C(y)} \mathrm{d} y=\frac{1}{d} \int_{0}^{1}(1+2 y)^{(2 a+b-3 d) / 3 d}(1-y)^{-(6 d+2 a-2 b) / 3 d} \mathrm{~d} y .
$$

Now by (6.4) we can easily obtain the conclusion that $J_{0}<\infty$ if and only if $3 d+r<2(b-a)$ and, hence, under the conditions that $C^{\prime}(1)=0$ and $B^{\prime}(1)>0$, we know that $a_{i}=1$ for 
all $i \geq 1$ if and only if $3 d+r \geq 2(b-a)$, which coincides with Corollary 4.3. Note that the case $3 d+r=2(b-a)$ corresponds to the case that $C^{\prime \prime}(1)=4 B^{\prime}(1)$ and, thus, by Corollary 4.3 we still have $a_{i}=1$ for all $i \geq 1$.

Finally, we consider the case $C^{\prime}(1)>0$, i.e. $c>2 d+r$, and, thus, $q<1$. For this case, if $q=a / b$, i.e. if $a / b=\left(d+r+\sqrt{(d+r)^{2}+4 d c}\right) / 2 c$ then $a_{i}=(a / b)^{i}(i \geq 1)$, while if $a<b q$ then the function $A(y)$ takes the form

$$
A(y)=\left(\frac{q-y}{q}\right)^{2(b q-a) / c(q-\zeta)}\left(\frac{y-\zeta}{-\zeta}\right)^{2(a-b \zeta) / c(q-\zeta)}
$$

We have obtained the following conclusion.

Theorem 6.1. For the IBC q-matrix determined by (6.1) and (6.2), we have the following conclusions.

(i) There always exists only one IBCP which is the Feller minimal process and this Feller minimal process is honest if and only if $c \leq 2 d+r$.

(ii) The extinction probabilities $a_{i}=1$ for all $i \geq 1$ if and only if one of the following three cases holds:

(a) $c<2 d+r$

(b) $c=2 d+r$ and $b \leq a$,

(c) $c=2 d+r, b>a$, and $3 d+r \geq 2(b-a)$.

(iii) If $c=2 d+r, b>a$, and $3 d+r<2(b-a)$, then $a_{i}<1$ and in this case the extinction probability $a_{i}$ is given by

$$
a_{i}=\frac{\int_{\zeta}^{1} y^{i}(1+(2+r / d) y)^{2(b d+a r+2 a d) /(3 d+r)(2 d+r)-1}(1-y)^{2(b-a) /(3 d+r)-2} \mathrm{~d} y}{\int_{\zeta}^{1}(1+(2+r / d) y)^{2(b d+a r+2 a d) /(3 d+r)(2 d+r)-1}(1-y)^{2(b-a) /(3 d+r)-2} \mathrm{~d} y},
$$

where $\zeta=-d /(2 d+r)$.

In particular, if $r=0$ then $a_{i}$ takes the particularly simply form

$$
a_{i}=\frac{\int_{-1 / 2}^{1} y^{i}(1+2 y)^{(2 a+b) / 3 d-1}(1-y)^{(2 b-2 a-6 d) / 3 d} \mathrm{~d} y}{\int_{-1 / 2}^{1}(1+2 y)^{(2 a+b) / 3 d-1}(1-y)^{(2 b-2 a-6 d) / 3 d} \mathrm{~d} y} .
$$

(iv) If $c>2 d+r, a_{i}<1$. Moreover, if $c>2 d+r$ and $a / b=\left(d+r+\sqrt{(d+r)^{2}+4 d c}\right) / 2 c$, then $a_{i}=(a / b)^{i}<1$, while if $c>2 d+r$ and $a / b<\left(d+r+\sqrt{(d+r)^{2}+4 d c}\right) / 2 c$, then

$$
a_{i}=\int_{\zeta}^{q} \frac{y^{i}(1-y / q)^{\alpha-1}(1-y / \zeta)^{\beta-1}}{1-y} \mathrm{~d} y / \int_{\zeta}^{q} \frac{(1-y / q)^{\alpha-1}(1-y / \zeta)^{\beta-1}}{1-y} \mathrm{~d} y
$$

where $q$ and $\zeta$ are given in the line below (6.3), and

$$
\alpha=\frac{2(b q-a)}{c(q-\zeta)} \quad \text { and } \quad \beta=\frac{2(a-b \zeta)}{c(q-\zeta)}
$$

are two positive constants. 
Remark 6.1. If one does the transformation $y=\zeta+(q-\zeta) x$ in (6.5) then the extinction probabilities $a_{i}$ can be rewritten as

$$
a_{i}=\frac{\sum_{k=0}^{i}\left(\begin{array}{l}
i \\
k
\end{array}\right)(q-\zeta)^{k} \zeta^{i-k} \int_{0}^{1}\left(\left(x^{k} x^{\beta-1}(1-x)^{\alpha-1}\right) /(u-x)\right) \mathrm{d} x}{\int_{0}^{1}\left(\left(x^{\beta-1}(1-x)^{\alpha-1}\right) /(u-x)\right) \mathrm{d} x},
$$

where $u=(1-\zeta) /(q-\zeta)>1$. In particular,

$$
a_{1}=\zeta+(q-\zeta) \int_{0}^{1} \frac{x^{\beta}(1-x)^{\alpha-1}}{(u-x)} \mathrm{d} x / \int_{0}^{1} \frac{x^{\beta-1}(1-x)^{\alpha-1}}{(u-x)} \mathrm{d} x .
$$

Note also that $a_{i}$ could be written as linear combinations of beta functions and, thus, gamma functions. This provides considerable convenience in calculating such extinction probabilities numerically.

Remark 6.2. Theorem 6.1(iv) is the same as that obtained in [10]. In fact, to the authors' knowledge, this result is the only result obtained for IBCPs until now. Note however that this result is a special example of the superexplosive case. Even for this very special example, the subtle subexplosive case remained unsolved until we tackled the problem. Using Theorem 5.9, we can easily write down the solution for the subexplosive case, but we will not do so here for obvious reasons.

Note that the extinction properties of IBCPs depend only on the two functions $B(s)$ and $C(s)$, and, conversely, if we know these two functions, we could easily obtain the original $Q$-matrix. For this reason, in the following examples, we will only specify these two functions rather than the $Q$-matrix itself.

Example 6.2. In this example $C^{\prime}(1)=+\infty$, but we keep $B(s)$ the same as in Example 6.1. That is, we consider an IBC $q$-matrix $Q$ to which the associated $B(s)$ and $C(s)$ are given by

$$
B(s)=a-(a+b) s+b s^{2}=(1-s)(a-b s)
$$

and

$$
C(s)=\frac{1}{4}+\frac{s}{2}-\frac{15}{8} s^{2}+\sum_{k=3}^{\infty}\left(a_{k-1}+\frac{a_{k}}{4}\right) s^{k}
$$

where $a, b>0$ and

$$
a_{k}=\left(1-\frac{1}{2(k-1)}\right) \frac{[2(k-2)] !}{\left[(k-2) ! 2^{k-2}\right]^{2}}, \quad k \geq 2 .
$$

Easy algebra shows that

$$
C(s)=\left(s+\frac{1}{4}\right)(1-s)\left[1-\frac{s}{\sqrt{1-s}}\right],
$$

and $B^{\prime}(1)=b-a$ and $C^{\prime}(1)=+\infty$. Also, $C(s)=0$ has three roots in $[-1,1]$, i.e. $-\frac{1}{4}, 1$, and $\rho_{c}=(\sqrt{5}-1) / 2$ (i.e. $\rho_{c}$ is the positive root of $s^{2}+s-1=0$ in $(0,1)$ ). By Theorem 3.1 we know that the corresponding IBCP is the dishonest Feller minimal process. Now, by the results obtained in the previous sections,

(i) if $a / b=\rho_{c}$ then $a_{i}=\rho_{c}{ }^{i}$,

(ii) if $a / b<\rho_{c}$ then

$$
a_{i}=\int_{-1 / 4}^{\rho_{c}} \frac{y^{i} A(y)}{C(y)} \mathrm{d} y / \int_{-1 / 4}^{\rho_{c}} \frac{A(y)}{C(y)} \mathrm{d} y,
$$


where

$$
A(y)=\exp \left\{\int_{0}^{y} \frac{2(a-b s)(1-s+s \sqrt{1-s})}{(1 / 4+s)\left(1-s-s^{2}\right)} \mathrm{d} s\right\} .
$$

Example 6.3. In this example we have $B^{\prime}(1)=+\infty$ together with an arbitrary structure of $C(s)$. Let $b_{0}=a>0, b_{2}=b / 2>0, b_{2 n}=b(2 n-3) ! ! /(2 n) ! !, n \geq 2, b_{2 n+1}=0$ for all $n \geq$ 1 , and $b_{1}=-(a+b)$. Then

$$
B(s)=a-(a+b) s+\frac{b}{2} s^{2}+b \sum_{n=2}^{\infty} \frac{(2 n-3) ! !}{(2 n) ! !} s^{2 n} .
$$

Easy algebra shows that $B(s)$ has the form

$$
B(s)=(1-s)\left[a+b-b \sqrt{\frac{1+s}{1-s}}\right],
$$

which has two positive zeros, 1 and $\rho_{b}$, where

$$
\rho_{b}=\frac{(a+b)^{2}-b^{2}}{(a+b)^{2}+b^{2}}
$$

together with the obvious fact that $B^{\prime}(1)=+\infty$. Furthermore, assume that $C(s)=\sum_{n=0}^{\infty} c_{n} s^{n}$ is in a general form with the zeros 1 and possibly $\rho_{c}$. Using the results obtained in the previous sections, we obtain the following result.

Theorem 6.2. Let $Q$ be an IBC $q$-matrix to which the associated $B(s)$ is given in (6.6) together with the general form of $C(s)=\sum_{n=0}^{\infty} c_{n} s^{n}$. Then we have the following conclusions.

(i) If $C^{\prime}(1)<0, a_{i}=1$.

(ii) If $0<C^{\prime}(1)<+\infty, a_{i}<1$. Moreover, if $\left((a+b)^{2}-b^{2}\right) /\left((a+b)^{2}+b^{2}\right)<\rho_{c}$,

$$
a_{i}=\int_{\zeta_{c}}^{\rho_{c}} \frac{y^{i} g(y) A(y)}{2(1-y)} \mathrm{d} x / \int_{\zeta_{c}}^{\rho_{c}} \frac{g(y) A(y)}{2(1-y)} \mathrm{d} y,
$$

where

$$
A(y)=\exp \left\{(a+b) \int_{0}^{y} g(x) \mathrm{d} x-b \int_{0}^{y} g(x) \sqrt{\frac{1+x}{1-x}} \mathrm{~d} x\right\},
$$

$g(y)=C(y) / 2(1-y)$ is a bounded and analytic function of $y$ on $[0,1]$, and $\zeta_{c} \in(-1,0)$ and $\rho_{c} \in(0,1)$ are zeros of $C(s)$.

Proof. By (6.6), it is easily seen that $B(s) \sim-2 b(1-s)^{1 / 2}$ (as $s \rightarrow 1^{-}$) and, thus, (i) immediately follows from Corollary 4.2 and the fact that $C^{\prime}(1)<0$. Part (ii) follows from Theorem 5.3 directly.

Finally, we provide an example in which the structures of $B(s)$ and $C(s)$ are very similar. We are interested to see what the effects are when $B(s)$ and $C(s)$ 'compete' in some sense.

Example 6.4. Let $Q$ be an IBC $q$-matrix to which the associated $B(s)$ and $C(s)$ are given by

$$
B(s)=a-(a+b) s+b(1-p) s^{2} \sum_{k=0}^{\infty}(p s)^{k}
$$


and

$$
C(s)=d-(d+c) s^{2}+c(1-q) s^{3} \sum_{k=0}^{\infty}(q s)^{k},
$$

where $a, b, c$, and $d$ are positive numbers, and $0<p<1$ and $0<q<1$. Easy algebra shows that

$$
B(s)=\frac{(1-s)[a-(a p+b) s]}{1-p s}=(1-s)\left[a-\frac{b s}{1-p s}\right]
$$

and that

$$
C(s)=(1-s)\left[d+d s-\frac{c s^{2}}{1-q s}\right] \quad \text { or } \quad C(s)=\frac{(d q+c)(1-s)\left(\rho_{c}-s\right)(s-\zeta)}{1-q s}
$$

with

$$
\rho_{c}=\frac{d(1-q)+\sqrt{d^{2}(1-q)^{2}+4 d(d q+c)}}{2(d q+c)}
$$

and

$$
\zeta=\frac{d(1-q)-\sqrt{d^{2}(1-q)^{2}+4 d(d q+c)}}{2(d q+c)} .
$$

By (6.9) we know that

$$
\rho_{b}=\frac{a}{a p+b}
$$

and that

$$
B^{\prime}(1)=\frac{b}{1-p}-a
$$

thus, $B^{\prime}(1)>0$ if and only if $1-p<b / a$.

The following key quantities can be easily calculated:

$$
C^{\prime}(1)=\frac{c}{1-q}-2 d \quad \text { and } \quad C^{\prime \prime}(1)=4 c-2 d+\frac{2 c q(3-2 q)}{(1-q)^{2}}<\infty .
$$

Under the condition that $C^{\prime}(1)=0, C^{\prime \prime}(1)$ can be further simplified as

$$
C^{\prime \prime}(1)=\frac{c(3-q)}{(1-q)^{2}} .
$$

Now applying the results obtained before to this example we easily obtain the following result.

Theorem 6.3. For the IBCP determined by the IBC $q$-matrix $Q$ associated with (6.7) and (6.8), we have the following conclusions.

(i) $Q$ is regular, i.e. the IBCP is honest if and only if $1-q \geq c / 2 d$.

(ii) For all $i \geq 1$, the extinction probabilities $a_{i}=1$ if and only if one of the following conditions holds:

(a) $1-q>c / 2 d$,

(b) $1-q=c / 2 d$ and $1-p \geq b / a$,

(c) $1-q=c / 2 d, 1-p<b / a$, and $(3-q) / 4(1-q)^{2}+a / c \geq b / c(1-p)$. 
(iii) If $1-q=c / 2 d, 1-p<b / a$, and $(3-q) / 4(1-q)^{2}+a / c<b / c(1-p)$, then

$$
a_{i}=\int_{\zeta}^{1} \frac{y^{i}(1-q y) A(y)}{(1-y)^{2}(y-\zeta)} \mathrm{d} y / \int_{\zeta}^{1} \frac{(1-q y) A(y)}{(1-y)^{2}(y-\zeta)} \mathrm{d} y,
$$

where

$$
A(y)=\exp \left\{\frac{2}{d q+c} \int_{0}^{y} \frac{(1-q x)[a-(a p+b) x]}{(1-p x)(1-x)(x-\zeta)} \mathrm{d} y\right\}
$$

and $\zeta$ is given in (6.11).

(iv) Suppose that $1-q<c / 2 d$. Then, if $\rho_{c}=\rho_{b}$,

$$
a_{i}=\left(\frac{a}{a p+b}\right)^{i}, \quad i \geq 1,
$$

while, if $\rho_{b}<\rho_{c}$,

$$
a_{i}=\int_{\zeta}^{\rho_{c}} \frac{y^{i}(1-q y) A(y)}{(1-y)\left(\rho_{c}-y\right)(y-\zeta)} \mathrm{d} y / \int_{\zeta}^{\rho_{c}} \frac{(1-q y) A(y)}{(1-y)\left(\rho_{c}-y\right)(y-\zeta)} \mathrm{d} y,
$$

where

$$
A(y)=\exp \left\{\frac{2}{d q+c} \int_{0}^{y} \frac{(1-q x)[a-(a p+b) x]}{(1-p x)\left(\rho_{c}-x\right)(x-\zeta)} \mathrm{d} x\right\}
$$

and $\rho_{c}$ and $\zeta$ are given in (6.10) and (6.11), respectively.

For this example, we are particularly interested in the so-called 'perfectly competitive' situation in the sense that $p=q, a=d$, and $b=c$. For this special case, we first note that $C^{\prime}(1)=0$ implies that both $B^{\prime}(1)=a>0$ and $C^{\prime \prime}(1)>4 B^{\prime}(1)$, and, thus, the extinction probabilities $a_{i}=1$ for all $i \geq 1$ so long as $C^{\prime}(1)=0$. Secondly, $C^{\prime}(1)>0$ implies that $B^{\prime}(1)>0$ and, thus, $\rho_{b}<1$. Indeed, $C^{\prime}(1)>0$ is equivalent to $b /(1-p)>2 a$, but $B^{\prime}(1)$ is just $b /(1-p)-a$. Moreover, we can further show that $C^{\prime}(1)>0$ implies that $0<\rho_{b}<\rho_{c}<1$. In fact, easy algebra shows that $\rho_{b}<\rho_{c}$ is equivalent to the trivial fact that $4 a b>0$. Now we obtain the following satisfactory conclusion, which shows that in the evolution of this IBCP the collision component totally dominates the branching component.

Corollary 6.1. For the IBCP process given in Example 6.4 with the further conditions that $0<p=q<1, a=d>0$, and $b=c>0$, the following statements are equivalent.

(i) The IBC q-matrix is regular, i.e. the IBCP is honest.

(ii) The extinction probabilities are all 1 , i.e. $a_{i}=1$ for all $i \geq 1$.

(iii) $1-p \geq b / 2 a$.

Furthermore, if $1-p<b / 2 a$ then the IBCP is dishonest and the extinction probability starting from $i \geq 1$ is less than 1 and these extinction probabilities are given by

$$
\begin{aligned}
a_{i}= & \int_{\zeta}^{\rho_{c}} \frac{y^{i}(1-p y) A(y)}{(1-y)\left[a(1+y)(1-p y)-b y^{2}\right]} \mathrm{d} y \\
& \times\left(\int_{\zeta}^{\rho_{c}} \frac{(1-p y) A(y)}{(1-y)\left[a(1+y)(1-p y)-b y^{2}\right]} \mathrm{d} y\right)^{-1},
\end{aligned}
$$


where

$$
A(y)=\exp \left\{2 \int_{0}^{y} \frac{a(1-p x)-b x}{a(1+x)(1-p x)-b x^{2}} \mathrm{~d} x\right\},
$$

and $\rho_{c}$ and $\zeta$ are the positive and negative roots of the equation $a(1+y)(1-p y)-b y^{2}=0$, respectively.

\section{Acknowledgements}

The authors would like to express their sincere thanks to the anonymous referees who provided extremely helpful comments and suggestions, which led to a much improved version of this paper. The work of Li Junping was substantially supported by grants from both the Research Fund for the Doctoral Program of Higher Education of China (grant number 20110162110060) and the National Natural Sciences Foundation of China (grant number 11071259).

\section{References}

[1] Anderson, W. J. (1991). Continuous-Time Markov Chains. Springer, New York.

[2] Asmussen, S. and Hering, H. (1983). Branching Processes. Birkhäuser, Boston, MA.

[3] Athreya, K. B. ANd Jagers, P. (eds) (1972). Classical and Modern Branching Processes. Springer, Berlin.

[4] Athreya, K. B. And Ney, P. E. (1983). Branching Processes. Springer, New York.

[5] Chen, A., Pollett, P., Li, J. and Zhang, H. (2010). Uniqueness, extinction and explosivity of generalised Markov branching processes with pairwise interaction. Methodology Comput. Appl. Prob. 12, 511-531.

[6] Chen, A., Pollett, P., Zhang, H. and Li, J. (2004). The collision branching process. J. Appl. Prob. 41, 1033-1048.

[7] Chen, M. F. (1992). From Markov Chains to Nonequilibrium Particle Systems. World Scientific, River Edge, NJ.

[8] Harris, T. E. (1963). The Theory of Branching Processes. Springer, Berlin.

[9] Kalinkin, A. V. (2002). Markov branching processes with interaction. Russian Math. Surveys 57, 241-304.

[10] Kalinkin, A. V. (2003). On the extinction probability of a branching process with two kinds of interaction of particles. Theory Prob. Appl. 46, 347-352.

[11] Lange, A. M. (2007). On the distribution of the number of final particles in a branching processe with transformations and pairwise interactions. Theory Prob. Appl. 51, 704-714.

[12] Li, J. AND Chen, A. (2006). Markov branching processes with immigration and resurrection. Markov Process. Relat. Fields 12, 139-168.

[13] Sevastyanov, B. A. And Kalinkin, A. V. (1982). Random branching processes with interaction of particles. Sov. Math. Dokl. 25, 644-648. 